\title{
FEEDBACK CONTROL FOR RANDOM, LINEAR HYPERBOLIC BALANCE LAWS
}

\author{
Markus Bambach, ${ }^{1}$ Stephan Gerster, ${ }^{2, *}$ Michael Herty, ${ }^{2} \mathcal{E}$ \\ Muhammad Imran ${ }^{3}$
}

\author{
${ }^{1}$ Institut für virtuelle Produktion, TH Zürich, Technoparkstraße 1, 8005 Zürich, Switzerland \\ ${ }^{2}$ Institut für Geometrie und Praktische Mathematik, RWTH Aachen University, \\ Templergraben 55, 52062 Aachen, Germany \\ ${ }^{3}$ Lehrstuhl Konstruktion und Fertigung, BTU Cottbus-Senftenberg, \\ Konrad-Wachsmann-Allee 17, 03046 Cottbus, Germany \\ *Address all correspondence to: Stephan Gerster, Institut für Geometrie und Praktische Mathematik, RWTH \\ Aachen University, Templergraben 55, 52062 Aachen, Germany, E-mail: gerster@igpm.rwth-aachen.de
}

\begin{abstract}
We design boundary controls of physical systems that are faced by uncertainties. The system dynamics are described by random hyperbolic balance laws. The control aims to steer the system to a desired state under uncertainties. We propose a control based on Lyapunov stability analysis of a suitable series expansion of the random dynamics. The control damps the impact of uncertainties exponentially fast in time. The presented approach can be applied to a large class of physical systems and random perturbations, as e.g. Gaussian processes. We illustrate the boundary control effect on a stochastic viscoplastic material model.
\end{abstract}

KEY WORDS: Systems of hyperbolic balance laws, feedback stabilization, Lyapunov function, stochastic Galerkin, viscoplastic deformations

\section{INTRODUCTION}

Boundary stabilization has been studied intensively in the past years [1]. A well-known approach to prove exponential stability of a desired state is the construction of suitable Lyapunov functionals and the analysis of so-called dissipative boundary conditions. Exponential decay of a continuous Lyapunov function under dissipative boundary conditions has been proven in [1-5]. Also explicit decay rates for numerical schemes have been established [6-10].

Most results are based on the assumption that model parameters and desired states are known exactly. However, often there is need to take uncertainties into account. For instance, model parameters are uncertain due to noisy measurements and due to variations in the behaviour of materials. Moreover, epistemic uncertainties arise, when the considered mathematical models do not exactly describe the true physics as e.g. in constitutive equations for material models.

When the underlying model is not known exactly, but is given by a probability law or by statistical moments, the deterministic stabilization concept should be extended to this stochastic case. MonteCarlo methods may be used to apply deterministic stabilization concepts to each realization. For instance, sampling-based methods are used in $[11,12]$ to analyze the existence of optimal solutions for some optimization problems with probabilistic constraints.

In contrast, the underlying tool used in this paper is the representation of stochastic perturbations by a series of orthogonal functions, known as generalized polynomial chaos (gPC) expansions [13-15]. Expansions of the stochastic input are substituted into the governing equations and they are projected by a Galerkin method to obtain deterministic evolution equations for the coefficients of this expansion. This intrusive 
approach is often applied in uncertainty quantification. In this direction many results for kinetic equations are available [16-22]. Recently, also results for hyperbolic equations have been established [23-30].

The problem if the random solutions to kinetic equations converge to the deterministic kinetic equilibrium exponentially fast has been analyzed [20,31-33] by using hypocoercivity properties [34-38]. However, results for stabilizing general hyperbolic systems are only partial. Already in the deterministic case solutions to hyperbolic conservation laws exist in the classical sense only in finite time due to the occurence of shocks [39] and Lyapunov's indirect method [40] does not necessarily hold. Furthermore, the results are so far restricted in the sense that the destabilizing effect of the source term is sufficiently small $[1,41,42]$. Explicit decay rates for the $L^{2}$-stabilization of steady states have been established in the simplified case of linear, deterministic systems [1, Prop. 5.2], which include Maxwell's, elasticity and linearized Euler equations.

This paper extends these results to the stochastic case. The stochastic system is reformulated as a sequence of deterministic problems. A weighted $L^{2}$-norm of this series is used as Lyapunov function. We will show that a modification of the dissipativity condition [2, Th. 2.3] yields an exponentially fast decaying Lyapunov function, which in turn makes the mean and variance of stochastic deviations diminish exponentially fast over time.

The presented approach is related to the variance reduction by robust design of boundary conditions [43]. There, energy estimates are derived to obtain well-posed boundary value problems and boundary conditions that reduce the variance in the system. In extension, the Lyapunov stability analysis [1,2] damps deviations exponentially fast over time as long as stronger assumptions on the boundary conditions and the source term are satisfied.

This paper is structured as follows. Section 2 is devoted to the representation of stochastic processes with orthogonal functions. We derive a deterministic formulation of the underlying stochastic boundary value problem. Section 3 considers a stability analysis for general linear hyperbolic balance laws. A Lyapunov function that gives an upper bound on deviations from desired states is presented. Furthermore, conditions on the boundary control are stated that make the Lyapunov functions and hence the deviations decay exponentially fast over time. Possible extensions to nonlinear systems are discussed in Section 4. Finally, Section 5 illustrates the presented approach by steering a viscoplastic material to a desired state under uncertainties.

\section{RANDOM HYPERBOLIC BOUNDARY VALUE PROBLEMS}

Uncertainties are included by a possibly multidimensional random variable $\xi: \Omega \rightarrow \mathbb{R}^{M}$ that is defined on a probability space $(\Omega, \mathcal{F}(\Omega), \mathbb{P})$. The space- and time-depending dynamics $y(t, x ; \xi(\omega)) \in \mathbb{R}^{2}$ are for each fixed realization $\omega \in \Omega$ described by $2 \times 2$ strictly hyperbolic balance laws of the form

$$
\partial_{t} y(t, x ; \xi(\omega))+A(x ; \xi(\omega)) \partial_{x} y(t, x ; \xi(\omega))=-S(x ; \xi(\omega)) y(t, x ; \xi(\omega)) \quad \text { for } \quad y=(v, \sigma)^{\mathrm{T}} .
$$

The matrix $A(x ; \xi(\omega)) \in \mathbb{R}^{2 \times 2}$, which describes advection, is assumed diagonalizable, Lipschitz-continuous and the source term $S(x ; \xi(\omega)) \in \mathbb{R}^{2 \times 2}$ is assumed continuous in $x \in[0, L]$. A typical model, which we consider in Section 5 , is a viscoplastic deformation described by a displacement velocity or acceleration $v$ and stress $\sigma$. If a linear control $B_{y} \in \mathbb{R}^{2 \times 2}$ is applied at the boundaries of the spatial domain $[0, L]$, the desired control is of the form

$$
\left(\begin{array}{c}
v(t, 0 ; \xi) \\
v(t, L ; \xi)
\end{array}\right)=B_{y}\left(\begin{array}{c}
\sigma(t, 0 ; \xi) \\
\sigma(t, L ; \xi)
\end{array}\right) .
$$

Various choices of the control $B_{y}$ are possible and a suitable choice will be presented in Section 5.1. In particular, we are interested in the control of deviations from a desired state. We introduce the notation

$$
\Delta y(t, x ; \xi):=y(t, x ; \xi)-y^{*}(x ; \xi),
$$

where $y^{*}(x ; \xi)$ denotes the desired state, which may include also uncertainties. The analysis is described for dynamics on a one-dimensional physFical domain $[0, L] \subset \mathbb{R}$, i.e. one arc. Dynamics $y^{(j)}=\left(v^{(j)}, \sigma^{(j)}\right)^{\mathrm{T}}$ 
on multiple arcs $j=1, \ldots, n$ can be coupled by specifying appropriate node conditions, as illustrated in Figure 1.

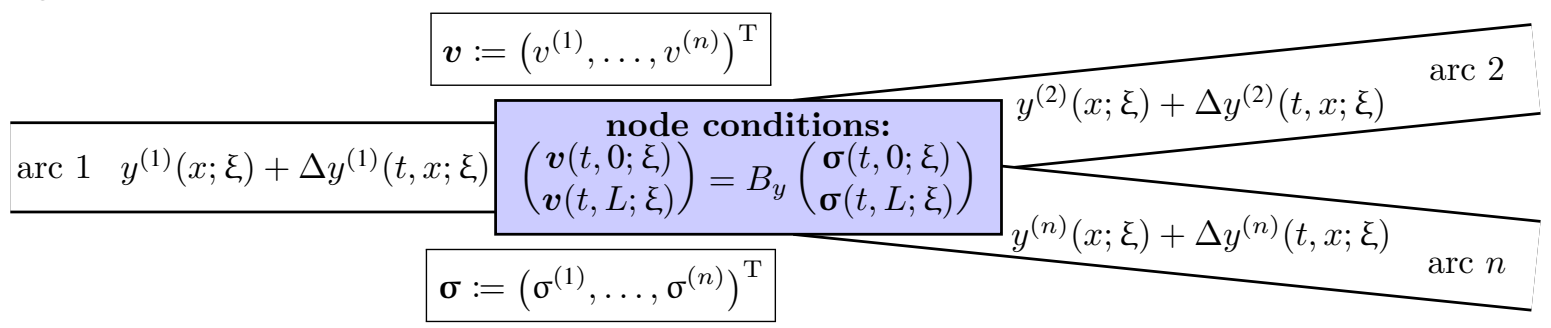

Figure 1: Network with $n$ arcs in a perturbed steady state with linear boundary conditions $B_{y} \in \mathbb{R}^{2 n \times 2 n}$.

A stabilization strategy could be to apply deterministic concepts to each possible realization. This "worstcase" ansatz, however, depends sensitively on possibly large deviation in the realizations. A more efficient stabilization concept is a mean squared error $\mathbb{E}\left[\|\Delta y(t, x ; \xi)\|^{2}\right]$ that should decay over time as fast as possible. Although negligibly unlikely events are permitted, the stabilization concept is still strong. It makes both the mean $\mathbb{E}$ and the variance $\mathbb{V}$ of deviations decay, since we have

$$
\mathbb{E}\left[\|\Delta y(t, \cdot ; \xi)\|^{2}\right]=\mathbb{E}[\|\Delta y(t, \cdot ; \xi)\|]^{2}+\mathbb{V}[\|\Delta y(t, \cdot ; \xi)\|]
$$

for any suitable norm $\|\cdot\|$. In the following we will consider, unless otherwise stated, the $L^{2}$-norm $\|\cdot\|_{L_{2}}$ with respect to space $x \in[0, L]$, i.e.

$$
\mathbb{E}\left[\|\Delta y(t, \cdot ; \xi)\|_{L^{2}}^{2}\right]=\mathbb{E}\left[\int \Delta v(t, x ; \xi)^{2}+\Delta \sigma(t, x ; \xi)^{2} \mathrm{~d} x\right] \quad \text { with } \quad \Delta y=\left(\begin{array}{c}
\Delta v \\
\Delta \sigma
\end{array}\right) .
$$

Furthermore, this stabilization concept allows for an equivalent representation of the mean squared error (3) by a series of deterministic equations, which will be introduced in this section.

\subsection{Representation of stochastic processes}

We consider a stochastic process $\operatorname{Pr} \in L^{2}((0, L) ; \mathbb{R}) \otimes \mathbb{L}^{2}(\Omega, \mathbb{P})$ that admits a tensor product structure such that for each fixed point in space $x \in(0, L)$ the process belongs to the $\mathbb{L}^{2}$-space

$$
\begin{aligned}
& \mathbb{L}^{2}(\Omega, \mathbb{P}):=\left\{\operatorname{Pr}: \Omega \rightarrow L^{2}((0, L) ; \mathbb{R}), \omega \mapsto \operatorname{Pr}(\cdot ; \omega) \mid\|\operatorname{Pr}(x ; \cdot)\|_{\mathbb{P}}<\infty\right\} \\
& \text { for } \quad\left\langle\operatorname{Pr}\left(x_{1} ; \cdot\right), \operatorname{Pr}\left(x_{2} ; \cdot\right)\right\rangle_{\mathbb{P}}:=\int \operatorname{Pr}\left(x_{1} ; \omega\right) \operatorname{Pr}\left(x_{2} ; \omega\right) \mathrm{d} \mathbb{P}(\omega) \\
& \text { and } \quad\|\operatorname{Pr}(x ; \cdot)\|_{\mathbb{P}}:=\sqrt{\langle\operatorname{Pr}(x ; \cdot), \operatorname{Pr}(x ; \cdot)\rangle_{\mathbb{P}}} .
\end{aligned}
$$

This tensor product structure allows to express the expected value and the covariance kernel by

$$
\begin{aligned}
& \mathbb{E}\left[\operatorname{Pr}\left(x_{1} ; \omega\right) \operatorname{Pr}\left(x_{2} ; \omega\right)\right]:=\left\langle\operatorname{Pr}\left(x_{1} ; \cdot\right), \operatorname{Pr}\left(x_{2} ; \cdot\right)\right\rangle_{\mathbb{P}}, \\
& \mathbb{C}\left(x_{1}, x_{2}\right):=\operatorname{Cov}\left[\operatorname{Pr}\left(x_{1} ; \omega\right), \operatorname{Pr}\left(x_{2} ; \omega\right)\right]:=\mathbb{E}\left[\operatorname{Pr}\left(x_{1} ; \omega\right) \operatorname{Pr}\left(x_{2} ; \omega\right)\right]-\mathbb{E}\left[\operatorname{Pr}\left(x_{1} ; \omega\right)\right] \mathbb{E}\left[\operatorname{Pr}\left(x_{2} ; \omega\right)\right]
\end{aligned}
$$

A centered stochastic process that is mean square continuous, i.e. $\left\|\operatorname{Pr}(x ; \cdot)-\operatorname{Pr}\left(x^{*} ; \cdot\right)\right\|_{\mathbb{P}} \rightarrow 0$ for $x \rightarrow x^{*}$, admits the Karhunen-Loève expansion

$$
\mathcal{K}[\operatorname{Pr}](x ; \omega):=\sum_{k=1}^{M} \sqrt{d_{k}} \psi_{k}(x) \xi_{k}(\omega) \quad \text { with } \quad \xi_{k}(\omega):=\frac{1}{\sqrt{d_{k}}} \int_{0}^{L} \operatorname{Pr}(x ; \omega) \psi_{k}(x) \mathrm{d} x,
$$


where $\psi_{k}$ and $d_{k}>0$ are eigenfunctions and eigenvalues of the covariance kernel [44]. Although the random variables $\xi_{k}$ are formally defined, there is in general no practical expression. For Gaussian processes, however, they are given by independent normally distributed random variables [44,45]. Therefore, Gaussian processes are often used, although it might be problematic, when representing bounded physical processes.

Time-varying stochastic dynamics cannot be expressed directly in terms of Karhunen-Loève expansions, which require that the covariance structure is known a priori. For our random dynamics, however, this structure is given only implicitly by a hyperbolic boundary value problem. To generalize the KarhunenLoève expansion, we introduce a generalized polynomial chaos (gPC) as a set of orthogonal subspaces

$$
\widehat{\mathcal{S}}_{k} \subseteq \mathbb{L}^{2}(\Omega, \mathbb{P}) \quad \text { with } \quad \mathcal{S}_{K}:=\bigoplus_{k=0}^{K} \widehat{\mathcal{S}}_{k} \rightarrow \mathbb{L}^{2}(\Omega, \mathbb{P}) \quad \text { for } \quad K \rightarrow \infty .
$$

These subspaces are spanned by orthogonal functions. Common choices for one-dimensional random variables $\xi(\omega) \in \mathbb{R}$ are the following polynomials $[15,45]$ :

Legendre polynomials $\quad$ with uniform distribution $\xi \sim \mathcal{U}(-1,1)$

$\phi_{0}(\xi)=1, \quad \phi_{1}(\xi)=\xi, \quad \phi_{k+1}(\xi)=\frac{2 k+1}{k+1} \xi \phi_{k}(\xi)-\frac{k}{k+1} \phi_{k-1}(\xi)$

Hermite polynomials with Gaussian distribution $\xi \sim \mathcal{N}(0,1)$

$\phi_{0}(\xi)=1, \quad \phi_{1}(\xi)=\xi, \quad \phi_{k+1}(\xi)=\xi \phi_{k}(\xi)-k \phi_{k-1}(\xi)$

Orthogonal functions with respect to multi-dimensional random parameters $\xi(\omega) \in \mathbb{R}^{M}$ can be constructed by introducing a multi-index $\boldsymbol{k}:=\left(k_{1}, \ldots, k_{M}\right)^{\mathrm{T}} \in \mathbb{K}$ associated to an index set $\mathbb{K} \subseteq \mathbb{N}_{0}^{M}$. Common choices are the index sets

$$
\begin{array}{llll}
\mathbb{K}=\left\{\boldsymbol{k} \in \mathbb{N}_{0}^{M} \mid\|\boldsymbol{k}\|_{0} \leq K\right\} & \text { for } & |\mathbb{K}|=(K+1)^{M}, \\
\mathbb{K}=\left\{\boldsymbol{k} \in \mathbb{N}_{0}^{M} \mid\|\boldsymbol{k}\|_{1} \leq K\right\} & \text { for } & |\mathbb{K}|=\frac{(M+K) !}{M ! K !}
\end{array}
$$

Since the random variables $\xi_{1}, \ldots, \xi_{M}$ are independent, the resulting polynomials

$$
\phi_{\boldsymbol{k}}(\xi):=\phi_{k_{1}}\left(\xi_{1}\right) \cdot \ldots \cdot \phi_{k_{M}}\left(\xi_{M}\right) \quad \text { satisfy } \quad\left\langle\phi_{\boldsymbol{i}}, \phi_{\boldsymbol{j}}\right\rangle_{\mathbb{P}}=\left\|\phi_{\boldsymbol{i}}\right\|_{\mathbb{P}}^{2} \delta_{\boldsymbol{i}, \boldsymbol{j}},
$$

where we write with abuse of notation $\left\langle\phi_{i}, \phi_{j}\right\rangle_{\mathbb{P}}=\left\langle\phi_{i}(\xi), \phi_{j}(\xi)\right\rangle_{\mathbb{P}}$. Then, a dynamic stochastic process $\operatorname{Pr}(t, x ; \xi)$ is approximated by an orthogonal projection

$$
\Pi_{\mathbb{K}}[\operatorname{Pr}](t, x ; \xi):=\sum_{\boldsymbol{k} \in \mathbb{K}} \widehat{\operatorname{Pr}}_{\boldsymbol{k}}(t, x) \phi_{\boldsymbol{k}}(\xi) \quad \text { with gPC modes } \widehat{\operatorname{Pr}}_{\boldsymbol{k}}(t, x):=\frac{\left\langle\operatorname{Pr}(t, x ; \cdot), \phi_{\boldsymbol{k}}(\cdot)\right\rangle_{\mathbb{P}}}{\left\|\phi_{\boldsymbol{k}}\right\|_{\mathbb{P}}^{2}}
$$

The truncated expansion $(\mathbb{K g P C})$ converges in the sense $\left\|\Pi_{\mathbb{K}}[\operatorname{Pr}](t, x ; \cdot)-\operatorname{Pr}(t, x ; \cdot)\right\|_{\mathbb{P}} \rightarrow 0$ for $K \rightarrow \infty$ provided that the probability measure satisfies mild conditions, which hold for Legendre and Hermite polynomials [14,46-48].

\subsection{Stochastic Galerkin formulation}

The underlying system (1) is linear and hence the random fluctuations also satisfy the differential equation

$$
\partial_{t} \Delta y(t, x ; \xi)+A(x ; \xi) \partial_{x} \Delta y(t, x ; \xi)=-S(x ; \xi) \Delta y(t, x ; \xi) .
$$


The eigenvalue decomposition

$$
A(x ; \xi)=T(x ; \xi) \Lambda(x ; \xi) T^{-1}(x ; \xi) \quad \text { with } \quad \Lambda(x ; \xi)=\operatorname{diag}\left\{\lambda^{+}(x ; \xi), \lambda^{-}(x ; \xi)\right\}
$$

allows to rewrite this system in Riemann coordinates

$$
\mathcal{R}(t, x ; \xi):=\left(\begin{array}{l}
\mathcal{R}^{+}(t, x ; \xi) \\
\mathcal{R}^{-}(t, x ; \xi)
\end{array}\right):=T^{-1}(x ; \xi) \Delta y(t, x ; \xi) .
$$

Then, the linear balance law (4) with boundary conditions (2) is equivalent to the boundary value problem

$$
\begin{aligned}
& \partial_{t} \mathcal{R}(t, x ; \xi)+\Lambda(x ; \xi) \partial_{x} \mathcal{R}(t, x ; \xi)=-C(x ; \xi) \mathcal{R}(t, x ; \xi) \\
& \left(\begin{array}{l}
\mathcal{R}^{+}(t, 0 ; \xi) \\
\mathcal{R}^{-}(t, L ; \xi)
\end{array}\right)=B\left(\begin{array}{l}
\mathcal{R}^{+}(t, L ; \xi) \\
\mathcal{R}^{-}(t, 0 ; \xi)
\end{array}\right)
\end{aligned}
$$

where the source term is defined as

$$
C(x ; \xi):=T^{-1}(x ; \xi) S(x ; \xi) T(x ; \xi)+\Lambda(x ; \xi) T^{-1}(x ; \xi) \partial_{x} T(x ; \xi) .
$$

The boundary control is described by a matrix $B$, which states the transformed boundary conditions from equation (2). We refer the reader to [8, Th. 6.1] for more details on this transform and we will state in Section 5.1 an illustrative example. Similarly to Figure 1, where the boundary control $B_{y}$ is stated in physical quantities, Figure 2 illustrates that the boundary value problem (7) is extendable to multiple arcs.

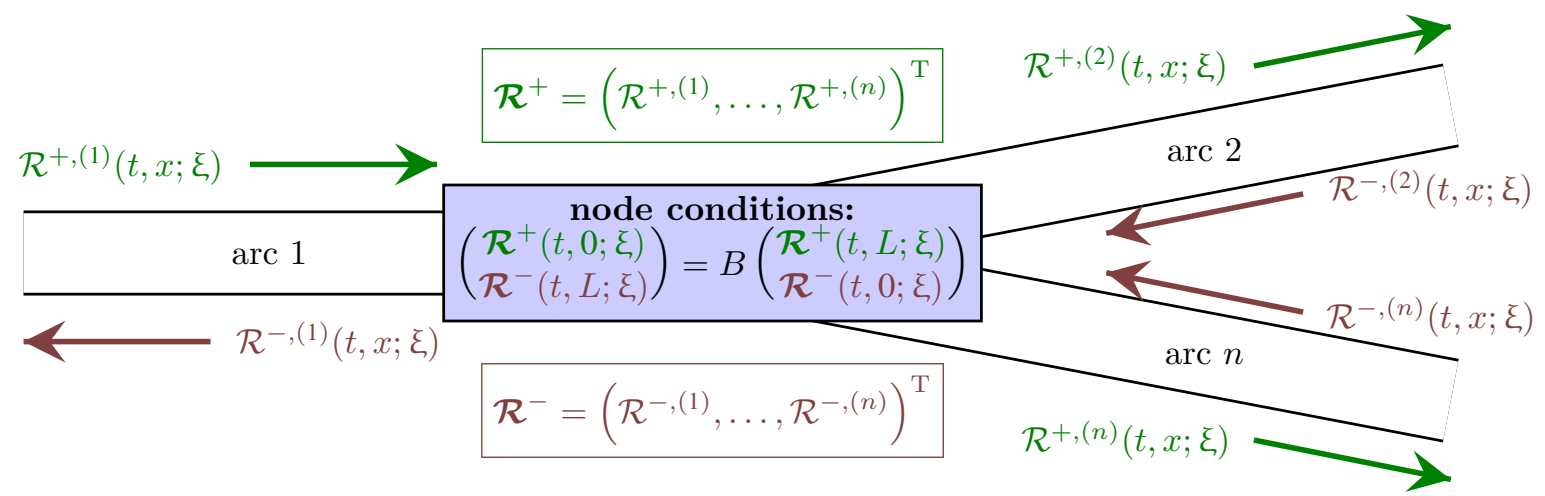

Figure 2: Network with $n$ arcs in Riemann coordinates with linear boundary conditions $B \in \mathbb{R}^{2 n \times 2 n}$.

We note that the eigenvalue decomposition (5) must satisfy

$$
\lambda^{-}(x ; \xi)<0<\lambda^{+}(x ; \xi) \text { for all } x \in[0, L] \text { and } \xi \sim \mathbb{P}
$$

such that the boundary control is applicable. In the following, we will transfer this property to a stochastic Galerkin formulation that expresses the random system (7) as a sequence of deterministic problems. To this end, we replace all random quantities by the gPC approximation $(\mathbb{K g P C})$, i.e.

$$
\begin{aligned}
& \lambda^{ \pm}(x ; \xi) \approx \Pi_{\mathbb{K}}\left[\lambda^{ \pm}\right](x ; \xi)=\sum_{k \in \mathbb{K}} \widehat{\lambda}_{\boldsymbol{k}}^{ \pm}(x) \phi_{\boldsymbol{k}}(\xi), \\
& C(x ; \xi) \quad \approx \Pi_{\mathbb{K}}[C](x ; \xi)=\sum_{\boldsymbol{k} \in \mathbb{K}} \widehat{\boldsymbol{C}}_{\boldsymbol{k}}(x) \phi_{\boldsymbol{k}}(\xi), \\
& \mathcal{R}(t, x ; \xi) \approx \Pi_{\mathbb{K}}[\mathcal{R}](t, x ; \xi)=\sum_{\boldsymbol{k} \in \mathbb{K}} \widehat{\mathcal{R}}_{\boldsymbol{k}}(t, x) \phi_{\boldsymbol{k}}(\xi) .
\end{aligned}
$$


The random system is projected onto the gPC basis $\left(\phi_{\boldsymbol{k}}\right)_{\boldsymbol{k} \in \mathbb{K}}$ by the Galerkin method

$$
\left\langle\partial_{t} \Pi_{\mathbb{K}}[\mathcal{R}](t, x ; \cdot)+\Pi_{\mathbb{K}}[\Lambda](x ; \cdot) \partial_{x} \Pi_{\mathbb{K}}[\mathcal{R}](t, x ; \cdot)+\Pi_{\mathbb{K}}[C](x ; \cdot) \Pi_{\mathbb{K}}[\mathcal{R}](t, x ; \cdot), \phi_{\boldsymbol{k}}(\cdot)\right\rangle_{\mathbb{P}}=0 \quad \text { for all } \quad \boldsymbol{k} \in \mathbb{K} .
$$

This leads to the stochastic Galerkin formulation

$$
\begin{aligned}
& \partial_{t} \widehat{\boldsymbol{\mathcal { R }}}(t, x)+\widehat{\boldsymbol{A}}(x) \partial_{x} \widehat{\boldsymbol{\mathcal { R }}}(t, x)=-\widehat{\boldsymbol{S}}(x) \widehat{\boldsymbol{\mathcal { R }}}(t, x) \quad \text { for } \quad \widehat{\boldsymbol{A}}:=\left(\begin{array}{ll}
\widehat{\boldsymbol{A}}^{+} & \\
& \widehat{\boldsymbol{A}}^{-}
\end{array}\right) \\
& \text {with } \quad \widehat{\boldsymbol{A}}^{ \pm}(x):=\sum_{\boldsymbol{k} \in \mathbb{K}} \widehat{\lambda}_{\boldsymbol{k}}^{ \pm}(x)\left(\left\langle\phi_{\boldsymbol{k}}, \phi_{\boldsymbol{i}} \phi_{\boldsymbol{j}}\right\rangle_{\mathbb{P}}\right)_{\boldsymbol{i}, \boldsymbol{j} \in \mathbb{K}} \\
& \text { and } \quad \widehat{\boldsymbol{S}}(x):=\sum_{\boldsymbol{k} \in \mathbb{K}} \widehat{\boldsymbol{C}}_{\boldsymbol{k}}(x)\left(\left\langle\phi_{\boldsymbol{k}}, \phi_{\boldsymbol{i}} \phi_{\boldsymbol{j}}\right\rangle_{\mathbb{P}}\right)_{\boldsymbol{i}, \boldsymbol{j} \in \mathbb{K}} \text {. }
\end{aligned}
$$

Since the matrices $\widehat{A}^{ \pm}(x)$ are symmetric, there exists an orthogonal eigenvalue decomposition

$$
\widehat{\boldsymbol{A}}^{ \pm}(x)=\widehat{\mathcal{T}}^{ \pm}(x) \widehat{\mathcal{D}}^{ \pm}(x) \widehat{\mathcal{T}}^{ \pm}(x)^{\mathrm{T}}
$$

This allows to diagonalize the stochastic Galerkin formulation (9) by introducing the second class of Riemann invariants

$$
\widehat{\zeta}(t, x)=\widehat{\mathcal{T}}(x)^{\mathrm{T}} \widehat{\mathcal{R}}(t, x) \quad \text { for } \quad \widehat{\mathcal{T}}(x):=\operatorname{diag}\left\{\widehat{\mathcal{T}}^{+}(x), \widehat{\mathcal{T}}^{-}(x)\right\}
$$

These Riemann invariants are described by the boundary value problem

$$
\begin{aligned}
& \partial_{t} \widehat{\boldsymbol{\zeta}}(t, x)+\widehat{\mathcal{D}}(x) \partial_{x} \widehat{\boldsymbol{\zeta}}(t, x)=-\widehat{\mathcal{Q}}(x) \widehat{\zeta}(t, x), \\
& \left(\begin{array}{c}
\widehat{\zeta}^{+}(t, 0) \\
\widehat{\zeta}^{-}(t, L)
\end{array}\right)=\widehat{\mathcal{B}}\left(\begin{array}{c}
\widehat{\zeta}^{+}(t, L) \\
\widehat{\zeta}^{-}(t, 0)
\end{array}\right) \\
& \text { for } \quad \widehat{\mathcal{B}}:=\left(\begin{array}{ll}
\widehat{\mathcal{T}}^{+}(0) & \\
& \widehat{\mathcal{T}}^{-}(L)
\end{array}\right)^{\mathrm{T}}\left(\begin{array}{ll}
B_{1,1} \mathbb{I} & B_{1,2} \mathbb{I} \\
B_{2,1} \mathbb{I} & B_{2,2} \mathbb{I}
\end{array}\right)\left(\begin{array}{ll}
\widehat{\mathcal{T}}^{+}(L) & \\
& \widehat{\mathcal{T}}^{-}{ }_{(0)}
\end{array}\right) \text {, }
\end{aligned}
$$

where $\mathbb{I} \in \mathbb{R}^{|\mathbb{K}| \times|\mathbb{K}|}$ denotes the identity matrix. The characteristic speeds and the source term read as

$$
\widehat{\mathcal{D}}(x):=\operatorname{diag}\left\{\widehat{\mathcal{D}}^{+}(x), \widehat{\mathcal{D}}^{-}(x)\right\} \quad \text { and } \quad \widehat{\mathcal{Q}}(x):=\widehat{\mathcal{T}}(x)^{\mathrm{T}} \widehat{\boldsymbol{C}}(x) \widehat{\mathcal{T}}(x)+\widehat{\mathcal{D}}(x) \widehat{\mathcal{T}}(x)^{\mathrm{T}} \partial_{x} \widehat{\mathcal{T}}(x)
$$

Note that solutions to the systems (1) and (4) can also be expanded in terms of polynomial chaos expansions. However, it has been observed in [26, Sec. 4.2] that the basis $\left(\mathbb{K}_{S}\right)$ may lead to a loss of hyperbolicity. In contrast, the stochastic Galerkin formulation (9) is always hyperbolic. To ensure the wellposedness of the boundary value problem (11), we have to guarantee

$$
\widehat{\mathcal{D}}^{-}(x)<0<\widehat{\mathcal{D}}^{+}(x) \text { for all } \quad x \in[0, L] .
$$

Indeed, [49, Th. 2] and [25, Th. 2.1] state that property (8) implies (12). Namely, the matrices $\widehat{\boldsymbol{A}}^{ \pm}$are strictly positive and negative definite, respectively, since for all $\widehat{y} \neq(0, \ldots, 0)^{\mathrm{T}}$ and basis functions $\phi_{\boldsymbol{k}}$ we have

$$
\widehat{y}^{\mathrm{T}} \widehat{\boldsymbol{A}}^{ \pm} \widehat{y}= \pm \int\left(\sqrt{\left|\Pi_{\mathbb{K}}\left[\lambda^{ \pm}\right](x ; \xi)\right|} \sum_{\boldsymbol{k} \in \mathbb{K}} \widehat{y}_{\boldsymbol{k}} \phi_{\boldsymbol{k}}(\xi)\right)^{2} \mathrm{~d} \mathbb{P} .
$$


The wellposedness of the boundary value problem (11) follows now directly from [1, Th. A.4]. More precisely, $\left[1\right.$, Th. A.4] states that the $L^{2}$-solution $\widehat{\zeta}: \mathbb{R}_{0}^{+} \times(0, L) \rightarrow \mathbb{R}^{2|\mathbb{K}|}$ to the boundary value problem $(11)$ with initial values $\widehat{\zeta}(0, \cdot) \in L^{2}\left((0, L) ; \mathbb{R}^{2|\mathbb{K}|}\right)$ is a continuous map

$$
\widehat{\zeta} \quad: \quad \mathbb{R}_{0}^{+} \rightarrow L^{2}\left((0, L) ; \mathbb{R}^{2|\mathbb{K}|}\right), \quad t \mapsto \widehat{\zeta}(t, \cdot)
$$

that is unique and that depends also continuously on initial data. Due to the underlying tensor product structure and the relation $(10)$, i.e. $\widehat{\mathcal{R}}(t, x)=\widehat{\mathcal{T}}(x) \widehat{\zeta}(t, x)$, we can interpret the gPC expansion

$$
\Pi_{\mathbb{K}}[\mathcal{R}] \quad: \quad \mathbb{R}_{0}^{+} \rightarrow L^{2}\left((0, L) ; \mathbb{R}^{2}\right) \bigotimes \mathbb{L}^{2}(\Omega, \mathbb{P}), \quad t \mapsto \Pi_{\mathbb{K}}[\mathcal{R}](t, \cdot ; \cdot)=\sum_{\boldsymbol{k} \in \mathbb{K}} \widehat{\mathcal{R}}_{\boldsymbol{k}}(t, \cdot) \phi_{\boldsymbol{k}}(\cdot)
$$

as a well-defined random $L^{2}$-solution.

random boundary value problem $(7)$ time $t$

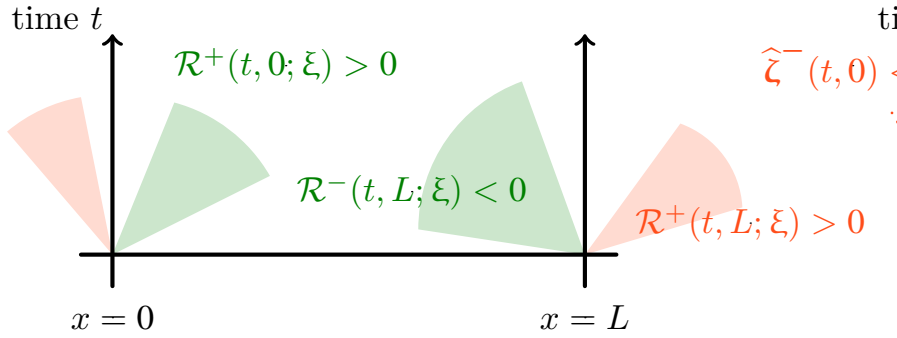

$$
\left(\begin{array}{l}
\mathcal{R}^{+}(t, 0 ; \xi) \\
\mathcal{R}^{-}(t, L ; \xi)
\end{array}\right)=B\left(\begin{array}{l}
\mathcal{R}^{+}(t, L ; \xi) \\
\mathcal{R}^{-}(t, 0 ; \xi)
\end{array}\right)
$$

deterministic boundary value problem (11)

time $t$

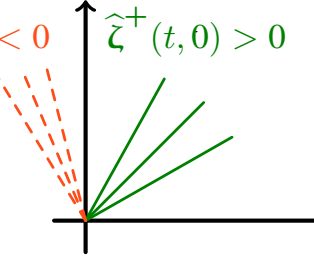

$x=0$

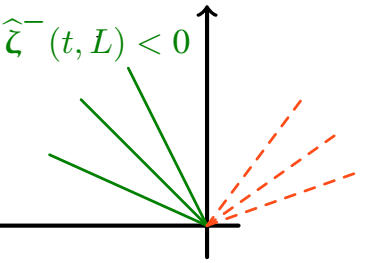

$\left(\begin{array}{c}\hat{\zeta}^{+}(t, 0) \\ \hat{\zeta}^{-}(t, L)\end{array}\right)=\widehat{\mathcal{B}}\left(\begin{array}{l}\hat{\zeta}^{+}(t, L) \\ \hat{\zeta}^{-}(t, 0)\end{array}\right)$

Figure 3: Random (left) and deterministic (right) boundary value problems (7) and (11) for $|\mathbb{K}|=3$. Characteristic speeds that point into the spatial domain are shown in green and those pointing out are shown in red, respectively.

Figure 3 summarizes the previous analysis in terms of specified boundary conditions. The left panel shows for the random boundary value problem (7) the domain of possible realizations of the characteristic curves, along which the realizations $\mathcal{R}^{ \pm}(t, x ; \xi(\omega))$ are constant. The domain corresponding to those curves that point into the spatial domain and influence the solution is shown in green. The domain corresponding to those pointing out is shown in red, respectively. Likewise, the deterministic solution $\widehat{\zeta}(t, x)$ to the system $(11)$ is constant on the set of characteristic curves as illustrated in the right panel. The corresponding boundary conditions, illustrated below in Figure 3, specify the new values (green) that propagate from the boundary into the domain in terms of those that go out of the domain (red).

The consideration of all possible realizations gives rise to a domain, where characteristic curves are located. This poses a challenge in the analysis, since the feasibility of the boundary control must be ensured for all realizations [12] and an appropriate stabilization concept must be introduced for the random systems. This challenge can be partially circumvented through a concept based on a function space of view, which is motivated by the fact that the $L^{2}$-solutions (14) and (13) contribute to the mean squared error (3) the same information. The solution (13) results for $K \rightarrow \infty$ in infinite, but countably many characteristic curves. We will show in the following section that this allows for a deterministic stability analysis.

Remark 1. We remark that condition (8) on the random characteristic speeds is sufficient, but not necessary. Consider a polynomial chaos approximation $(\mathbb{K g P C})$ with Hermite polynomials and truncation $K=1$ 
that contains the Karhunen-Loève decomposition (KL) for a Gaussian process as special case [44,45], i.e.

$$
\Pi_{\mathbb{K}}\left[\lambda^{+}\right](x ; \xi(\omega))=\mathcal{K}\left[\lambda^{+}\right](x ; \omega)=\mathbb{E}\left[\lambda^{+}(x ; \xi)\right]+\sum_{k=1}^{M} \sqrt{d_{k}} \psi_{k}(x) \xi_{k}(\omega) .
$$

By exploiting the orthogonality $\left\langle\xi_{k}, \xi_{\ell}\right\rangle_{\mathbb{P}}=\delta_{k, \ell}$ and by using a lexicographic ordering [50, Ch. 5] of the multi-indices $\boldsymbol{k} \in \mathbb{K}$ we obtain the matrix $\widehat{\boldsymbol{A}}^{+}$in the stochastic Galerkin formulation (9) as

$$
\widehat{\boldsymbol{A}}^{+}:=\left(\begin{array}{cccc}
\mathbb{E}\left[\lambda^{+}(x ; \xi)\right] & \sqrt{d_{1}} \psi_{1}(x) & \cdots & \sqrt{d_{M}} \psi_{M}(x) \\
\sqrt{d_{1}} \psi_{1}(x) & \mathbb{E}\left[\lambda^{+}(x ; \xi)\right] & & \\
\vdots & & \ddots & \\
\sqrt{d_{M}} \psi_{M}(x) & & & \mathbb{E}\left[\lambda^{+}(x ; \xi)\right]
\end{array}\right) \in \mathbb{R}^{(M+1) \times(M+1)} .
$$

It is strictly positive definite according to Gershgorin circle theorem provided that

$$
\mathbb{E}\left[\lambda^{+}(x ; \xi)\right]>\sum_{k=1}^{M}\left|\sqrt{d_{k}} \psi_{k}(x)\right|
$$

is satisfied. This assumption is not restrictive if the deviations from the mean of the stochastic process are sufficiently small. We note that the mean and the variance are directly given by the gPC modes for normalized $g P C$ expansions as

$$
\begin{aligned}
\mathbb{E}\left[\Pi_{\mathbb{K}}\left[\lambda^{+}\right](x ; \xi)\right] & =\widehat{\lambda}_{\mathbb{O}}^{+}(x), \quad \mathbb{O}:=(0, \ldots, 0)^{\mathrm{T}} \\
\mathbb{V}\left[\Pi_{\mathbb{K}}\left[\lambda^{+}\right](x ; \xi)\right] & =\mathbb{E}\left[\Pi_{\mathbb{K}}\left[\lambda^{+}\right](x ; \xi)^{2}\right]-\mathbb{E}\left[\Pi_{\mathbb{K}}\left[\lambda^{+}\right](x ; \xi)\right]^{2} \\
& =\sum_{\boldsymbol{k}, \boldsymbol{\ell} \in \mathbb{K}} \widehat{\lambda}_{\boldsymbol{k}}^{+}(x) \widehat{\lambda}_{\ell}^{ \pm}(x)\left\langle\phi_{\boldsymbol{k}}, \phi_{\ell}\right\rangle_{\mathbb{P}}-\widehat{\lambda}_{\mathbb{O}}^{+}(x)^{2} \\
& =\sum_{\boldsymbol{k} \in \mathbb{K} \backslash\{\mathbb{O}\}} \widehat{\lambda}_{\boldsymbol{k}}^{+}(x)^{2} .
\end{aligned}
$$

Therefore, property (16) yields

$$
\mathbb{E}\left[\mathcal{K}\left[\lambda^{+}\right](x ; \omega)\right]>\sum_{k=1}^{M}\left|\sqrt{d_{k}} \psi_{k}(x)\right| \geq \sqrt{\sum_{k=1}^{M} d_{k} \psi_{k}^{2}(x)}=\mathbb{V}\left[\mathcal{K}\left[\lambda^{+}\right](x ; \omega)\right]^{1 / 2}
$$

and the coefficient of variation (CV) must satisfy

$$
\mathrm{CV}\left[\mathcal{K}\left[\lambda^{+}\right](x ; \omega)\right]:=\frac{\mathbb{V}\left[\mathcal{K}\left[\lambda^{+}\right](x ; \omega)\right]^{1 / 2}}{\mathbb{E}\left[\mathcal{K}\left[\lambda^{+}\right](x ; \omega)\right]}<1 \quad \text { for all } \quad x \in[0, L]
$$

In fact, the truncation $K<\infty$ acts as a dimension reduction that makes the necessary conditions less restrictive. However, we note that for sufficiently large $K \in \mathbb{N}_{0}$ the matrix $\widehat{\boldsymbol{A}}^{+}$has both positive and negative eigenvalues if the random variable $\Pi_{\mathbb{K}}\left[\lambda^{+}(x ; \xi)\right]$ has both positive and negative realizations [25, Th. 2.1]. Since the Gaussian distribution involves realizations on the whole real line, the $\gamma$-distribution has been proposed in [50,51] as a "truncated Gaussian model". Then, positivity can be ensured for all realizations and for all truncations $K \in \mathbb{N}_{0}$. 


\section{LYAPUNOV STABILITY ANALYSIS}

We design a boundary control to diminish the mean squared error (3) exponentially fast over time. Due to relation (6) it suffices to guarantee that the stochastic Riemann invariants decay exponentially fast, i.e.

$$
\mathbb{E}\left[\left\|\Pi_{\mathbb{K}}[\mathcal{R}](t, \cdot ; \xi)\right\|_{L^{2}}^{2}\right] \leq c e^{-\mu t} \mathbb{E}\left[\left\|\Pi_{\mathbb{K}}[\mathcal{R}](0, \cdot ; \xi)\right\|_{L^{2}}^{2}\right]
$$

with decay rate $\mu>0$ and positive constant $c>0$. By exploiting the orthogonality $\widehat{\mathcal{T}}^{\mathrm{T}}=\widehat{\mathcal{T}}^{-1}$ we obtain the relation

$$
\mathbb{E}\left[\left\|\Pi_{\mathbb{K}}[\mathcal{R}](t, \cdot ; \xi)\right\|_{L^{2}}^{2}\right]=\|\widehat{\mathcal{R}}(t, \cdot)\|_{L^{2}}^{2}=\|\widehat{\mathcal{T}}(\cdot) \widehat{\zeta}(t, \cdot)\|_{L^{2}}^{2}=\|\widehat{\zeta}(t, \cdot)\|_{L^{2}}^{2}
$$

where the last equality makes the $L^{2}$-norm essential. We observe that the stochastic stabilization follows from the deterministic stabilization of the augmented system (11). These quantities do not have a physical meaning. However, the derived control will be formulated in the original quantities, which leads to an explicit and implementable feedback control. The proof of the following theorem is a modification of the Lyapunov stability analysis in [1, Sec. 5].

Theorem 2. Define the weights $W(x):=\operatorname{diag}\left\{W^{+}(x), W^{-}(x)\right\}, W^{ \pm}(x):=\operatorname{diag}\left\{w_{1}^{ \pm}(x), \ldots, w_{|\mathbb{K}|}^{ \pm}(x)\right\}$ by

$$
\begin{aligned}
w_{k}^{+}(x) & :=\frac{h_{k}^{+}}{\widehat{\mathcal{D}}_{k}^{+}(x)} \exp \left(-\hat{\mu} \int_{0}^{x} \frac{1}{\widehat{\mathcal{D}}_{k}^{+}(s)} \mathrm{d} s\right), \\
w_{k}^{-}(x) & :=\frac{h_{k}^{-}}{\left|\widehat{\mathcal{D}}_{k}^{-}(x)\right|} \exp \left(\hat{\mu} \int_{x}^{L} \frac{1}{\widehat{\mathcal{D}}_{k}^{-}(s)} \mathrm{d} s\right)
\end{aligned}
$$

for $k=1, \ldots,|\mathbb{K}|$ and assume given positive values $\hat{\mu}, h_{k}^{+}, h_{k}^{-}>0$ that satisfy the dissipativity condition

$$
\begin{array}{ll}
e^{\hat{\mu} \frac{L}{2 \lambda \min }}\left\|\mathcal{D} \widehat{\mathcal{B}} \mathcal{D}^{-1}\right\|_{2} \leq 1 \\
\text { for } \quad \lambda_{\min }:=\min _{\substack{x \in[0, L], k=1, \ldots,|\mathbb{K}|}}\left\{\widehat{\mathcal{D}}_{k}^{+}(x),\left|\widehat{\mathcal{D}}_{k}^{-}(x)\right|\right\} \\
\text { and } & \mathcal{D}:=\operatorname{diag}\left\{h_{1}^{+}, \ldots, h_{|\mathbb{K}|}^{+}, h_{1}^{-}, \ldots, h_{|\mathbb{K}|}^{-}\right\} .
\end{array}
$$

Here, $\|A\|_{2}:=\sigma_{\max }\left\{A^{\mathrm{T}} A\right\}^{1 / 2}$ denotes the spectral norm of a matrix with largest absolute eigenvalue $\sigma_{\max } \cdot$ Then, the mean squared gPC expansion, corresponding to the boundary value problem (7), satisfies

$$
\begin{aligned}
& \mathbb{E}\left[\left\|\Pi_{\mathbb{K}}[\mathcal{R}](t, \cdot ; \xi)\right\|_{L^{2}}^{2}\right] \leq c e^{-\mu t} \mathbb{E}\left[\left\|\Pi_{\mathbb{K}}[\mathcal{R}](0, \cdot ; \xi)\right\|_{L^{2}}^{2}\right] \\
& \text { for } \quad \mu=\hat{\mu}+\min _{x \in[0, L]}\left\{\sigma_{\min }\left\{W(x) \widehat{\mathcal{Q}}(x)+\widehat{\mathcal{Q}}(x)^{\mathrm{T}} W(x)\right\}\right\},
\end{aligned}
$$

where $\sigma_{\text {min }}$ denotes the smallest eigenvalue of the space-varying, symmetric matrix $W \widehat{\mathcal{Q}}+\widehat{\mathcal{Q}}^{\mathrm{T}} W$. Proof (Similarly to [1, Sec. 5]). The weights (18) define the Lyapunov function

$$
\mathcal{L}(t):=\int_{0}^{L} \widehat{\zeta}(t, x)^{\mathrm{T}} W(x) \widehat{\zeta}(t, x) \mathrm{d} x .
$$


To prove its exponential decay, we define the matrices

$$
\begin{aligned}
& \mathcal{H}:=\widehat{\mathcal{B}}^{\mathrm{T}}\left(\begin{array}{ll}
W^{+}(0) \widehat{\mathcal{D}}^{+}(0) & \\
& W^{-}(L)\left|\widehat{\mathcal{D}}^{-}(L)\right|
\end{array}\right) \widehat{\mathcal{B}}-\left(\begin{array}{ll}
W^{+}(L) \widehat{\mathcal{D}}^{+}(L) & \\
& W^{-}(0)\left|\widehat{\mathcal{D}}^{-}(0)\right|
\end{array}\right), \\
& \mathcal{M}(x):=-\frac{\partial}{\partial x}(W(x) \widehat{\mathcal{D}}(x))+W(x) \widehat{\mathcal{Q}}(x)+\widehat{\mathcal{Q}}(x)^{\mathrm{T}} W(x) .
\end{aligned}
$$

It has been shown in $[8]$ that the matrix $\mathcal{H}$ is negative semidefinite if inequality $(\mathcal{D})$ holds. For now, we assume

$$
\widehat{\zeta} \in C^{1}\left([0, \infty) \times[0, L] ; \mathbb{R}^{2|\mathbb{K}|}\right) .
$$

Since the matrix $2 W \widehat{\mathcal{Q}}$ and the symmetric matrix $W \widehat{\mathcal{Q}}+\widehat{\mathcal{Q}}^{\mathrm{T}} W$ represent the same quadratic form, i.e.

$$
\widehat{\zeta}(t, x)^{\mathrm{T}} W(x) \widehat{\mathcal{Q}}(x) \widehat{\zeta}(t, x)=\widehat{\zeta}(t, x)^{\mathrm{T}} \frac{W(x) \widehat{\mathcal{Q}}(x)+\widehat{\mathcal{Q}}(x)^{\mathrm{T}} W(x)}{2} \widehat{\zeta}(t, x),
$$

the time derivative of the Lyapunov function is

$$
\begin{aligned}
\mathcal{L}^{\prime}(t)= & 2 \int_{0}^{L} \widehat{\zeta}(t, x)^{\mathrm{T}} W(x) \partial_{t} \widehat{\zeta}(t, x) \mathrm{d} x \\
= & -2 \int_{0}^{L} \widehat{\zeta}(t, x)^{\mathrm{T}} W(x) \widehat{\mathcal{D}}(x) \partial_{x} \widehat{\zeta}(t, x) \mathrm{d} x-2 \int_{0}^{L} \widehat{\zeta}(t, x)^{\mathrm{T}} W(x) \widehat{\mathcal{Q}}(x) \widehat{\zeta}(t, x) \mathrm{d} x \\
= & \int_{0}^{L} \widehat{\zeta}(t, x)^{\mathrm{T}}\left[\frac{\partial}{\partial x}(W(x) \widehat{\mathcal{D}}(x))-W(x) \widehat{\mathcal{Q}}(x)-\widehat{\mathcal{Q}}(x)^{\mathrm{T}} W(x)\right] \widehat{\zeta}(t, x) \mathrm{d} x \\
& -\left[\widehat{\zeta}(t, L)^{\mathrm{T}} W(L) \widehat{\mathcal{D}}(L) \widehat{\zeta}(t, L)-\widehat{\zeta}(t, 0)^{\mathrm{T}} W(0) \widehat{\mathcal{D}}(0) \widehat{\zeta}(t, 0)\right] .
\end{aligned}
$$

Using the linear feedback boundary conditions, the boundary terms (24) read as

$$
\begin{aligned}
& -\left[\widehat{\zeta}(t, L)^{\mathrm{T}} W(L) \widehat{\mathcal{D}}(L) \widehat{\zeta}(t, L)-\widehat{\zeta}(t, 0)^{\mathrm{T}} W(0) \widehat{\mathcal{D}}(0) \widehat{\zeta}(t, 0)\right] \\
& =\left(\begin{array}{cc}
\widehat{\zeta}^{+}(t, 0) \\
\widehat{\zeta}^{-}(t, L)
\end{array}\right)^{\mathrm{T}}\left(\begin{array}{ll}
W^{+}(0) \widehat{\mathcal{D}}^{+}(0) & \\
& -W^{-}(L) \widehat{\mathcal{D}}^{-}(L)
\end{array}\right)\left(\begin{array}{l}
\widehat{\zeta}^{+}(t, 0) \\
\widehat{\zeta}^{-}(t, L)
\end{array}\right) \\
& -\left(\begin{array}{ll}
\widehat{\zeta}^{+}(t, L) \\
\widehat{\zeta}^{-}(t, 0)
\end{array}\right)^{\mathrm{T}}\left(\begin{array}{ll}
W^{+}(L) \widehat{\mathcal{D}}^{+}(L) & \\
& -W^{-}(0) \widehat{\mathcal{D}}^{-}(0)
\end{array}\right)\left(\begin{array}{l}
\widehat{\zeta}^{+}(t, L) \\
\widehat{\zeta}^{-}(t, 0)
\end{array}\right) \\
& =\left(\begin{array}{l}
\widehat{\zeta}^{+}(t, L) \\
\widehat{\zeta}^{-}(t, 0)
\end{array}\right)^{\mathrm{T}} \mathcal{H}\left(\begin{array}{c}
\widehat{\zeta}^{+}(t, L) \\
\widehat{\zeta}^{-}(t, 0)
\end{array}\right) \text {. }
\end{aligned}
$$

We recall that $\sigma_{\min }$ denotes the smallest eigenvalue of a matrix and we recall that the matrix $\mathcal{H}$ is negative semidefinite. Then, we obtain the estimates

$$
\begin{aligned}
\mathcal{L}^{\prime}(t) & =\left(\begin{array}{c}
\widehat{\zeta}^{+}(t, L) \\
\widehat{\zeta}^{-}(t, 0)
\end{array}\right)^{\mathrm{T}} \mathcal{H}\left(\begin{array}{c}
\widehat{\zeta}^{+}(t, L) \\
\widehat{\zeta}^{-}(t, 0)
\end{array}\right)-\int_{0}^{L} \widehat{\zeta}(t, x)^{\mathrm{T}} \mathcal{M}(x) \widehat{\zeta}(t, x) \mathrm{d} x \\
& \leq-\min _{x \in[0, L]}\left\{\sigma_{\min }\left\{W^{-1 / 2}(x) \mathcal{M}(x) W^{-1 / 2}(x)\right\}\right\} \mathcal{L}(t) \\
\Longrightarrow \quad \mathcal{L}(t) & \leq e^{-\mu t} \mathcal{L}(0), \quad \mu:=\min _{x \in[0, L]}\left\{\sigma_{\min }\left\{W^{-1 / 2}(x) \mathcal{M}(x) W^{-1 / 2}(x)\right\}\right\} .
\end{aligned}
$$


Due to $\partial_{x}(W(x) \widehat{\mathcal{D}}(x))=-\hat{\mu} W(x)$ the guaranteed decay rate (19) is obtained.

So far, we have assumed differentiable solutions (23) that satisfy boundary conditions. By following the approach in [1, Sec. 2.1], the previous analysis can be extended to general $L^{2}$-solutions. Since initial values $\widehat{\zeta}(0, x) \in C^{1}\left((0, L) ; \mathbb{R}^{2|\mathbb{K}|}\right)$ are dense in $L^{2}\left((0, L) ; \mathbb{R}^{2|| \mathbb{K} \mid}\right)$, there is a differentiable sequence

$$
\widehat{\zeta}^{(j)}(0, x) \in C^{1}\left((0, L) ; \mathbb{R}^{2|\mathbb{K}|}\right)
$$

that converges to initial values $\widehat{\zeta}(0, x)$ in $L^{2}\left((0, L) ; \mathbb{R}^{2|\mathbb{K}|}\right)$. This sequence vanishes for $x=0, x=L$ and satisfies the linear boundary conditions. It has been shown in [1, Th. A.1] that also the solution for $t>0$ is differentiable and satisfies

$$
\widehat{\zeta}^{(j)} \in C^{1}\left([0, \infty) ; L^{2}\left((0, L)^{2|\mathbb{K}|}\right)\right) \bigcap C^{0}\left([0, \infty) ; H^{1}\left((0, L)^{2|\mathbb{K}|}\right)\right),
$$

where $H^{1}$ denotes a Sobolev space. This regularity is sufficient to obtain the sequence of estimates

$$
\mathcal{L}^{(j)}(t) \leq e^{-\mu t} \mathcal{L}^{(j)}(0) \quad \text { for } \quad \mathcal{L}^{(j)}(t):=\int_{0}^{L} \widehat{\zeta}^{(j)}(t, x)^{\mathrm{T}} W(x) \widehat{\zeta}^{(j)}(t, x) \mathrm{d} x
$$

which implies $\mathcal{L}(t) \leq e^{-\mu t} \mathcal{L}(0)$ for $j \rightarrow \infty$.

Finally, we state a sufficient condition to ensure the dissipativity condition, which can be verified analytically.

Corollary 1. The dissipativity condition $(\mathcal{D})$ holds if the boundary control B satisfies

$$
e^{\hat{\mu} \frac{L}{2 \lambda_{\min }}}\|B\|_{2} \leq 1 \quad \text { for } \quad \lambda_{\min }:=\min _{\substack{q=1, \ldots, Q, x \in[0, L]}}\left\{\Pi_{\mathbb{K}}\left[\lambda^{+}\right]\left(x ; \xi^{(q)}\right),\left|\Pi_{\mathbb{K}}\left[\lambda^{-}\right]\left(x ; \xi^{(q)}\right)\right|\right\},
$$

where $\xi^{(1)}, \ldots, \xi^{(Q)}$ denote $Q:=\left\lceil\frac{3}{2}(K+1)\right\rceil$ Gaussian quadrature nodes.

Proof. Since the orthogonal matrices $\widehat{\mathcal{T}}^{ \pm}$preserve the spectral matrix norm, we have $\|\widehat{\mathcal{B}}\|_{2}=\|B\|_{2}$. Due to the relation

$$
\widehat{\mathcal{T}}^{ \pm}(x) \widehat{\mathcal{D}}^{ \pm}(x) \widehat{\mathcal{T}}^{ \pm}(x)^{\mathrm{T}}=\widehat{\boldsymbol{A}}^{ \pm}(x)=\left(\left\langle\Pi_{\mathbb{K}}\left[\lambda^{ \pm}\right](x ; \cdot), \phi_{\boldsymbol{i}}(\cdot) \phi_{\boldsymbol{j}}(\cdot)\right\rangle_{\mathbb{P}}\right)_{\boldsymbol{i}, \boldsymbol{j} \in \mathbb{K}}
$$

the eigenvalues $\widehat{\mathcal{D}}^{ \pm}$coincide with those of the matrix containing the projected random eigenvalues. These are in turn within the range of the random spectrum of the random characteristic speeds [49, Th. 2]. The integrals in equation (25) are computed exactly with Gaussian quadrature nodes $\xi^{(q)}$ and weights $\widehat{w}^{(q)}$ as

$$
\begin{aligned}
\left\langle\Pi_{\mathbb{K}}\left[\lambda^{ \pm}\right](x ; \cdot), \phi_{i}(\cdot) \phi_{j}(\cdot)\right\rangle_{\mathbb{P}} & =\sum_{\boldsymbol{k} \in \mathbb{K}} \prod_{\ell=1}^{M} \widehat{\lambda}_{k_{\ell}}^{ \pm}(x) \quad\left\langle\phi_{k_{\ell}}\left(\xi_{\ell}\right), \phi_{i_{\ell}}\left(\xi_{\ell}\right) \phi_{j_{\ell}}\left(\xi_{\ell}\right)\right\rangle_{\mathbb{P}} \\
& =\sum_{\boldsymbol{k} \in \mathbb{K}} \prod_{\ell=1}^{M} \widehat{\lambda}_{k_{\ell}}^{ \pm}(x)\left[\sum_{q=1}^{Q} \phi_{k_{\ell}}\left(\xi^{(q)}\right) \phi_{i_{\ell}}\left(\xi^{(q)}\right) \phi_{j_{\ell}}\left(\xi^{(q)}\right) \widehat{w}^{(q)}\right] .
\end{aligned}
$$

Hence, the random characteristic speeds can be estimated by evaluating only at Gaussian quadrature nodes. 
Although the statement of Theorem 2 seems to be abstract, it is in fact quite practical. All quantities can be computed numerically. The control only influences the dissipativity condition $(\mathcal{D})$. It is a modification of the well-known deterministic dissipativity condition

$$
\rho_{2}(B):=\inf _{\mathcal{D} \in \mathbb{D}}\left\{\left\|\mathcal{D} B \mathcal{D}^{-1}\right\|_{2}\right\}<1
$$

where $\mathbb{D}$ denotes the set of strictly positive diagonal matrices [2, Sec. 2]. Corollary 1 allows for a practical verification of this condition.

\section{NONLINEAR HYPERBOLIC CONSERVATION LAWS}

The Lyapunov stability analysis in Section 3 is based on $L^{2}$-solutions [1, Appendix A] to linear hyperbolic balance laws. Extensions to nonlinear systems are only partial, since Lyapunov's indirect method [40] does not necessarily hold for hyperbolic systems, i.e. the exponential stability of a nonlinear hyperbolic system does not follow from the stability of its linearization [1, Sec. 4]. Furthermore, solutions to conservation laws exist in the classical sense only in finite time due to the occurence of shocks [39,52]. Hence, the stochastic Galerkin formulation should be performed for a conservative formulation. There are several attempts to deduce hyperbolic stochastic Galerkin formulations for nonlinear hyperbolic systems. We mention here briefly three approaches for shallow water equations [53], where the gPC modes $\hat{h}$ and $\hat{q}$ describe random water height and mass flux, as well as for isothermal Euler equations $[27,28]$ with unknown density $\hat{\rho}$. The parameters are the gravitational constant $g>0$ and the speed of sound $a>0$. Furthermore, the entropy closure [54] yields a hyperbolic stochastic Galerkin formulation for general systems that are endowed with a strictly convex entropy.

\section{Shallow water equations:}

$$
\begin{aligned}
& \partial_{t}\left(\begin{array}{c}
\hat{\rho}(t, x) \\
\hat{q}(t, x)
\end{array}\right)+\left(\begin{array}{c}
\hat{q}(t, x) \\
\mathcal{P}(\hat{q}(t, x)) \mathcal{P}^{-1}(\hat{\rho}(t, x)) \hat{\rho}(t, x)+\frac{g}{2} \mathcal{P}(\hat{\rho}(t, x)) \hat{\rho}(t, x)
\end{array}\right)=0 \\
& \text { for } \quad \mathcal{P}(\hat{\rho}):=\sum_{\boldsymbol{k} \in \mathbb{K}} \hat{\rho}_{\boldsymbol{k}}\left(\left\langle\phi_{\boldsymbol{k}}(\cdot), \phi_{\boldsymbol{i}}(\cdot) \phi_{\boldsymbol{j}}(\cdot)\right\rangle_{\mathbb{P}}\right)_{\boldsymbol{i}, \boldsymbol{j} \in \mathbb{K}}
\end{aligned}
$$

Roe variable transform for isothermal Euler equations:

$\partial_{t}\left(\begin{array}{c}\hat{\rho}(t, x) \\ \hat{q}(t, x)\end{array}\right)+\left(\begin{array}{c}\hat{q}(t, x) \\ \mathcal{P}(\hat{\beta}(t, x)) \hat{\beta}(t, x)+a^{2} \hat{\rho}(t, x)\end{array}\right)=0$

for $\quad \hat{\beta}:=\mathcal{P}^{-1}(\hat{\alpha}) \hat{q} \quad$ and $\quad \hat{\alpha}:=\underset{\tilde{\alpha}}{\operatorname{argmin}}\left\{\frac{\tilde{\alpha} \mathcal{P}(\tilde{\alpha}) \tilde{\alpha}}{3}-\tilde{\alpha}^{\mathrm{T}} \hat{\rho}\right\}$

\section{Entropy Closure:}

$\partial_{t}\left\langle y(t, x ; \cdot), \phi_{\boldsymbol{k}}(\cdot)\right\rangle_{\mathbb{P}}+\partial_{x}\left\langle f(y(t, x ; \cdot)), \phi_{\boldsymbol{k}}(\cdot)\right\rangle=0$

for $\quad \boldsymbol{k} \in \mathbb{K}$ and $y(t, x ; \xi):=\underset{\tilde{y}}{\operatorname{argmin}}\left\{\int \eta(\tilde{y}) \mathrm{d} \mathbb{P}\right\}$ such that $\left\langle\tilde{y}, \phi_{\boldsymbol{k}}(\cdot)\right\rangle_{\mathbb{P}}=\hat{y}_{\boldsymbol{k}}(t, x)$

All of these approaches yield for general gPC bases a hyperbolic system in conservative form

$$
\partial_{t} \hat{y}(t, x)+\partial_{x} \hat{f}(\hat{y}(t, x))=0 \quad \text { with diagonalizable Jacobian } \quad \mathrm{D}_{\hat{y}} \hat{f}(\hat{y})=\widehat{\mathcal{T}}(\hat{y}) \widehat{\mathcal{D}}(\hat{y}) \widehat{\mathcal{T}}^{-1}(\hat{y}) .
$$

Without loss of generality, ordered eigenvalues $\widehat{\Lambda}(\hat{y})=\operatorname{diag}\left\{\widehat{\Lambda}^{+}(\hat{y}), \widehat{\Lambda}^{-}(\hat{y})\right\}$ with $\widehat{\Lambda}^{-}(\hat{y})<0<\widehat{\Lambda}^{+}(\hat{y})$ are assumed. We introduce the notation $\hat{y}=\left(\hat{y}^{+}, \hat{y}^{-}\right)^{\mathrm{T}}$ with cardinality $\left|\widehat{\Lambda}^{ \pm}(\hat{y})\right|=\left|\hat{y}^{ \pm}\right|$. Then, the conservation 
law (27) can be endowed with the possibly nonlinear boundary conditions

$$
\left(\begin{array}{c}
\hat{y}^{+}(t, 0) \\
\hat{y}^{-}(t, L)
\end{array}\right)=\mathcal{B}_{\widehat{y}}\left(\begin{array}{c}
\hat{y}^{+}(t, L) \\
\hat{y}^{-}(t, 0)
\end{array}\right) \quad \text { for } \quad \mathcal{B}_{\widehat{y}} \in C^{2}\left(\mathbb{R}^{2|\mathbb{K}|}, \mathbb{R}^{2|\mathbb{K}|}\right)
$$

such that the boundary value problem (27) and (28) is well-posed [1, Appendix B]. According to [1, Sec. 1 and Sec. 4], a direct extension of the $L^{2}$-stability analysis in Section 3 would require a transform in Riemann coordinates, i.e. a diffeomorphism $\varphi(\hat{y})=\widehat{\zeta}$ satisfying

$$
\mathrm{D}_{\hat{y}} \varphi(\hat{y}) \mathrm{D}_{\hat{y}} \hat{f}(\hat{y})=\widehat{\mathcal{D}}(\hat{y}) \mathrm{D}_{\hat{y}} \varphi(\hat{y}) .
$$

Then, the system (27) is for $C^{1}$-solutions with norm

$$
\|\hat{y}(t, \cdot)\|_{C^{1}}:=\max _{x \in[0, L]}\left\{\|\hat{y}(t, x)\|_{0}+\left\|\partial_{x} \hat{y}(t, x)\right\|_{0}\right\}<\infty \quad \text { and } \quad\|\hat{y}(t, x)\|_{0}:=\max _{\boldsymbol{k} \in \mathbb{K}}\left\{\left|\hat{y}_{\boldsymbol{k}}(t, x)\right|\right\}
$$

equivalent to the diagonal form $\partial_{t} \widehat{\boldsymbol{\zeta}}+\widehat{\mathcal{D}}\left(\varphi^{-1}(\widehat{\zeta})\right) \partial_{x} \widehat{\zeta}=0$. The transform (6) for linear systems is a special case, i.e. $\varphi(\hat{y})=T^{-1} \hat{y}$. For general nonlinear systems, however, finding the change of coordinates $\varphi$ requires to solve the first-order differential equation (29). For systems of size $|\hat{y}| \geq 3$ the solution $\varphi$ exists only in specific cases $[1,55,56]$. This issue can be partially circumvented for $C^{1}$-solutions to conservation laws of the form (27) with constant steady states, where at least the Jacobian is diagonalizable. The following theorem states how boundary conditions can be specified such that $C^{1}$-solutions are stable.

Theorem 3 (According to [1, Def. 4.17 and Th. 4.18]). Assume boundary conditions (28) of the form

$$
\rho_{\infty}\left(\mathcal{B}_{\widehat{y}}^{\prime}\right):=\inf _{\mathcal{D} \in \mathbb{D}}\left\{\left\|\mathcal{D} \mathcal{B}_{\widehat{y}}^{\prime} \mathcal{D}^{-1}\right\|_{\infty}\right\}<1 \text { with Jacobian } \quad \mathcal{B}_{\widehat{y}}^{\prime}:=\left.\mathrm{D}_{\hat{y}} \mathcal{B}_{\widehat{y}}(\hat{y})\right|_{\hat{y}=\hat{y}^{*}} .
$$

Then, there are constants $\mu, \varepsilon>0$ such that

$$
\begin{aligned}
& \left\|\hat{y}(t, \cdot)-\hat{y}^{*}(\cdot)\right\|_{C^{1}} \lesssim e^{-\mu t}\left\|\hat{y}(0, \cdot)-\hat{y}^{*}(\cdot)\right\|_{C^{1}} \\
& \text { for all initial values } \hat{y}(0, \cdot) \in\left\{\hat{y}_{0} \in C^{1}\left([0, L] ; \mathbb{R}^{2|\mathbb{K}|}\right) \mid\left\|\hat{y}_{0}-\hat{y}^{*}\right\|_{C^{1}}<\varepsilon\right\} .
\end{aligned}
$$

Theorem 3 also allows to couple systems on different arcs, as illustrated in Figure 4.

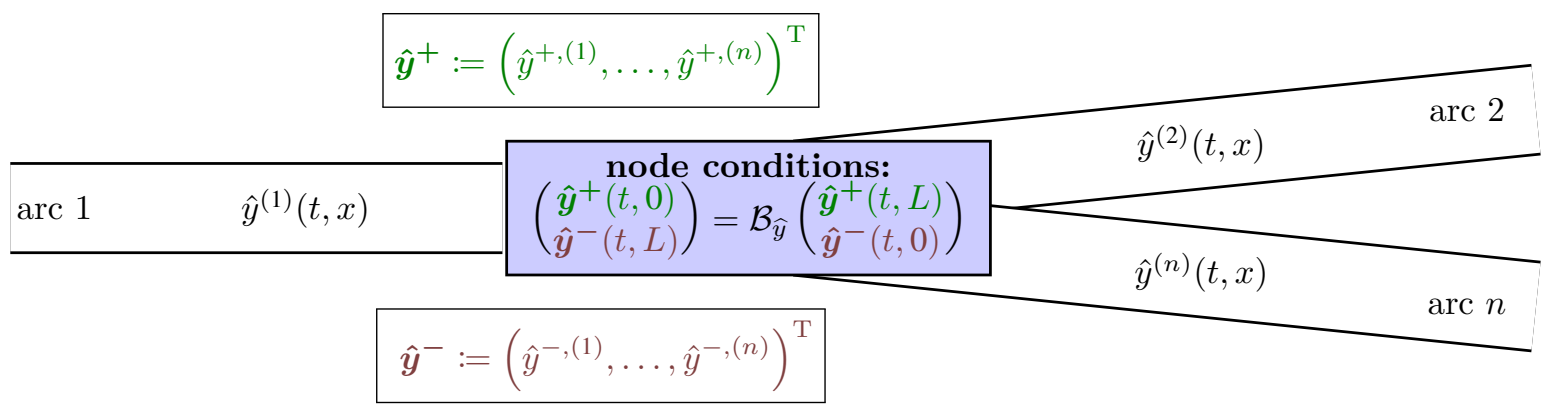

Figure 4: Network with $n$ arcs for dynamics described by the boundary value problem (27) and (28).

However in comparison to the stability analysis for linear systems with dissipativity condition (26), Theorem 3 does not account for source terms and the $C^{1}$-solution concept requires more restrictive assumptions on the boundary conditions [1, Prop. 4.7], since the inequality $\rho_{2}\left(\mathcal{B}_{\hat{y}}^{\prime}\right) \leq \rho_{\infty}\left(\mathcal{B}_{\hat{y}}^{\prime}\right)$ holds. Furthermore, Theorem 3 requires initial data to be close to a steady state. This makes well-balanced numerical schemes essential. We mention here briefly that well-balanced schemes have been developed for stochastic Galerkin formulations to scalar equations [57] and to shallow water equations [53]. Since they must be specifically adopted to a particular model, we limit ourselves in the following to linear systems. 


\section{ELASTIC AND VISCOPLASTIC DEFORMATIONS}

Elastic deformations are typically small displacements about a reference state [58, Sec. 2.12.1]. The displacements lead to strains, i.e. small deformations within the material. The interior forces because of stretching and compression of atomic bonds are called stress. These forces result in acceleration of the material and in turn affect the evolution of strains. Hence, the constitutive equations consist of Newton's law, relating stress and force to acceleration, and a stress-strain relationship. Viscoplastic deformations can be modelled by decomposing the total strain

$$
\epsilon(t, x)=\epsilon^{e}(t, x)+\epsilon^{p}(t, x)
$$

into an elastic part $\epsilon^{e}$ and plastic part $\epsilon^{p}$. There are several one-dimensional models that describe a displacement $u(t, x)$ and stress $\sigma(t, x)$ at time $t \geq 0$ and position $x \in[0, L]$. An overview on various models can be found e.g. in [59]. Here, the elastic relationship is described by

$$
\sigma(t, x)=E\left(\epsilon(t, x)-\epsilon^{p}(t, x)\right)
$$

with a constant $E>0$. A given stress-strain relationship allows to view the plastic part as a function of the stress, i.e. $\bar{\epsilon}^{p}(\sigma)$. Figure 5 shows two models and typical measurements in the left panel. A Bergström-model states a stress-strain relationship in terms of the ordinary differential equation

$$
\frac{\partial \rho(\epsilon)}{\partial \epsilon}=\frac{\dot{\epsilon} U_{0}}{T} \sqrt{\rho(\epsilon)}-\left(\Omega_{0}+C \exp \left(-\frac{m Q}{R T} \dot{\epsilon}^{-m}\right)\right) \rho(\epsilon), \quad \sigma(\epsilon)=\sigma_{0}+\alpha G b \sqrt{\rho(\epsilon)} .
$$

We refer the reader to [60], where a description of typical model parameters is given. This model is extended by the DRX-model that takes a softening due to dynamically recrystallized grains into account. If a critical stress $\epsilon_{c}>0$ is reached, the Bergström-model is replaced by

$$
\begin{aligned}
X(\varepsilon) & :=1-\exp \left(-\kappa\left(\frac{\epsilon-\epsilon_{c}}{\epsilon_{s}-\epsilon_{c}}\right)^{q}\right), \quad X_{\sigma}(\epsilon):=\int_{\epsilon_{c}}^{\epsilon} X^{\prime}(\epsilon) \sigma\left(\epsilon-\epsilon_{c}\right) \mathrm{d} \epsilon, \\
\sigma(\epsilon) & :=\sigma\left(\epsilon_{c}\right)(1-X(\epsilon))+X_{\sigma}(\epsilon) .
\end{aligned}
$$

Here, $X(\epsilon)$ is the recrystallized volume fraction and $\kappa, q>0$ are further parameters. We refer the reader to [61] for a more detailed description.
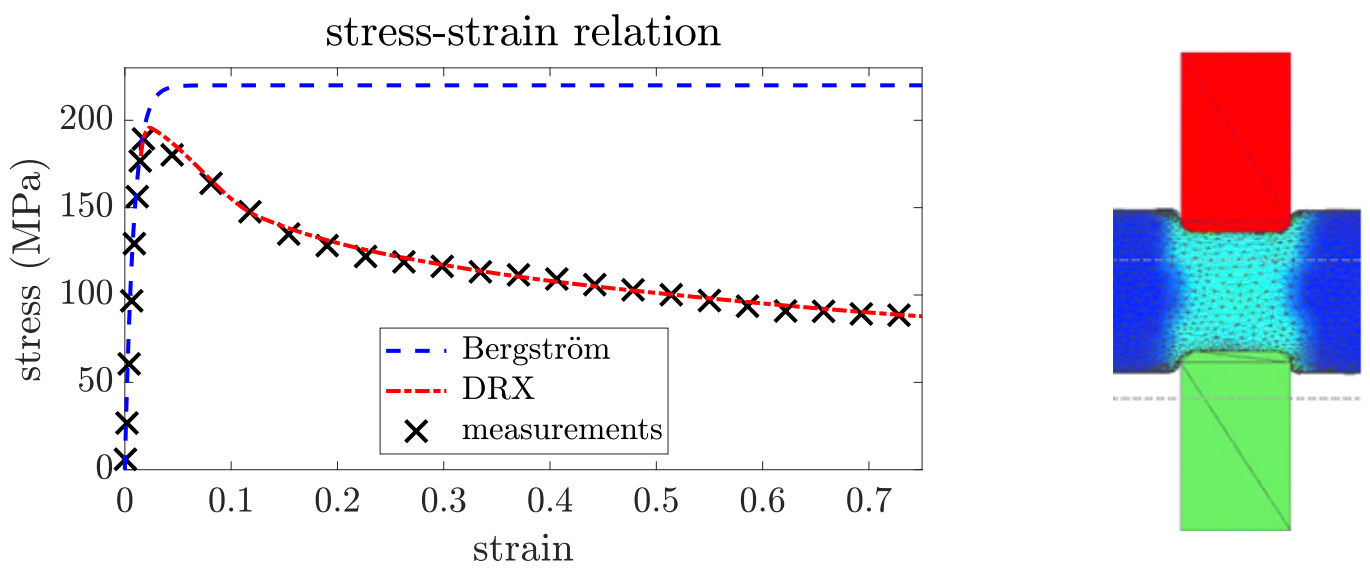

Figure 5: Left: Bergström- and DRX-model compared to sample measurements. Right: Sketch of a forming process. 
Then, the displacement velocity, i.e. the acceleration $v(t, x):=\partial_{t} u(t, x)$, and the stress are described by the balance law

$$
\frac{\partial}{\partial t}\left(\begin{array}{l}
v(t, x) \\
\sigma(t, x)
\end{array}\right)+\left(\begin{array}{cc} 
& -1 \\
-E &
\end{array}\right) \frac{\partial}{\partial x}\left(\begin{array}{l}
v(t, x) \\
\sigma(t, x)
\end{array}\right)=\left(\begin{array}{c}
0 \\
\bar{\epsilon}^{p}(\sigma(t, x))
\end{array}\right) .
$$

This model is used to describe a one-dimensional cross-section of a forming process as illustrated in the right panel of Figure 5.

\subsection{Derivation of a control rule}

Microstructural features such as grain size can be estimated from the stress. This is necessary to impose plastic deformations. The stress can be used as indicator for the microstructural states which cannot be measured during a forming process. Hence, there is an interest to control the system to a desired stress $\sigma^{*}$. We assume a source term that is linear close to the desired state, i.e. $\bar{\epsilon}^{p}(\sigma)=\bar{\epsilon}^{p}\left(\sigma^{*}\right)+\bar{\epsilon}_{\sigma}^{p}\left(\sigma^{*}\right) \Delta \sigma$ with a constant $\bar{\epsilon}_{\sigma}^{p}\left(\sigma^{*}\right):=\partial_{\sigma} \bar{\epsilon}^{p}\left(\sigma^{*}\right) \in \mathbb{R}$ that is obtained by a Taylor series approximation. Then, the deviations of the desired state are described by the linear system

$$
\begin{aligned}
& \partial_{t} \Delta y(t, x)+A \partial_{x} \Delta y(t, x)=-S(x) \Delta y(t, x) \\
& \text { with } A=\left(\begin{array}{rr}
-1 \\
-E
\end{array}\right) \text { and } S=-\left(\begin{array}{cc}
0 & 0 \\
0 & \bar{\epsilon}_{\sigma}^{p}\left(\sigma^{*}\right)
\end{array}\right) .
\end{aligned}
$$

In this particular example the advection part is uniform, but the source term is space-varying. We diagonalize the Jacobian

$$
A=T \Lambda T^{-1} \quad \text { with } \quad T=\left(\begin{array}{cc}
-1 & 1 \\
\sqrt{E} & \sqrt{E}
\end{array}\right) \text { and } \quad \Lambda=\left(\begin{array}{cc}
\sqrt{E} & \\
& -\sqrt{E}
\end{array}\right)
$$

to obtain the Riemann invariants and the corresponding source term as

$$
\mathcal{R}=\left(\begin{array}{c}
\mathcal{R}^{+} \\
\mathcal{R}^{-}
\end{array}\right):=T^{-1} \Delta y=\frac{1}{2}\left(\begin{array}{c}
\frac{\Delta \sigma}{\sqrt{E}}-\Delta v \\
\frac{\Delta \sigma}{\sqrt{E}}+\Delta v
\end{array}\right), \quad C=T^{-1} S T=-\frac{\bar{\epsilon}_{\sigma}^{p}\left(\sigma^{*}\right)}{2}\left(\begin{array}{ll}
1 & 1 \\
1 & 1
\end{array}\right) .
$$

For the system $\mathcal{R}_{t}+\Lambda \mathcal{R}_{x}=-C \mathcal{R}$ we prescribe the linear feedback boundary conditions

$$
\left(\begin{array}{c}
\mathcal{R}^{+}(t, 0) \\
\mathcal{R}^{-}(t, L)
\end{array}\right)=\left(\begin{array}{ll} 
& \kappa_{0} \\
\kappa_{1} &
\end{array}\right)\left(\begin{array}{l}
\mathcal{R}^{+}(t, L) \\
\mathcal{R}^{-}(t, 0)
\end{array}\right) .
$$

These boundary conditions, reformulated in terms of the displacement velocity, read as

$$
\begin{aligned}
& v(t, 0)=v^{*}(0)+\frac{1-\kappa_{0}}{\sqrt{E}+\kappa_{0} \sqrt{E}}\left(\sigma(t, 0)-\sigma^{*}(0)\right), \\
& v(t, L)=v^{*}(L)+\frac{\kappa_{1}-1}{\sqrt{E}+\sqrt{E} \kappa_{1}}\left(\sigma(t, L)-\sigma^{*}(L)\right),
\end{aligned} \quad \text { i.e. the control } B_{y}=\left(\begin{array}{ll}
\frac{1-\kappa_{0}}{\sqrt{E}+\kappa_{0} \sqrt{E}} & \frac{\kappa_{1}-1}{\sqrt{E}+\sqrt{E} \kappa_{1}}
\end{array}\right)
$$

for the linear boundary conditions (2) is obtained. Parameters $\kappa_{0}, \kappa_{1} \in \mathbb{R}$ should be specified such that the dissipativity condition $(\mathcal{D})$ in Theorem 2 holds. Furthermore, a large constant $\hat{\mu}>0$ is desirable, which leads to a larger decay rate $\mu$. For instance, the ansatz $\mu(\hat{\mu}) \geq 0, \exp \left(\hat{\mu} \frac{L}{2 \lambda_{\min }}\right)\|B\|=1$ yields

$$
\begin{aligned}
\mu(\hat{\mu}) & =\hat{\mu}-\left|\frac{\bar{\epsilon}_{\sigma}^{p}\left(\sigma^{*}\right)}{2}\right| \max _{x \in[0, L]}\left\{3 e^{-\frac{\hat{\mu} x}{\sqrt{E}}}+e^{-\frac{\hat{\mu}(L-x)}{\sqrt{E}}}, e^{-\frac{\hat{\mu} x}{\sqrt{E}}}+3 e^{-\frac{\hat{\mu}(L-x)}{\sqrt{E}}}\right\} \\
& \geq \hat{\mu}-2\left|\bar{\epsilon}_{\sigma}^{p}\left(\sigma^{*}\right)\right|=0 \quad \text { for } \quad \hat{\mu}:=2\left|\bar{\epsilon}_{\sigma}^{p}\left(\sigma^{*}\right)\right| .
\end{aligned}
$$

With this choice, the dissipativity condition is satisfied for $\kappa_{i}=\kappa:=e^{\frac{L}{\sqrt{E}}\left|\bar{\epsilon}_{\sigma}^{p}\left(\sigma^{*}\right)\right|}$ and the feedback law (30) reads as

$$
v(t, 0)=v^{*}(0)+\frac{1}{\sqrt{E}} \operatorname{coth}\left(\frac{L}{\sqrt{E}}\left|\bar{\epsilon}_{\sigma}^{p}\left(\sigma^{*}\right)\right|\right)\left(\sigma(t, 0)-\sigma^{*}(0)\right) .
$$




\subsection{Gaussian random fields}

Uncertainties due to unknown model parameters and uncertain steady states can be described by Gaussian random fields. These are completely characterized by their mean and covariance kernel. To be precise, for any given $n \in \mathbb{N}$, all locations $\vec{x}:=\left(x_{1}, \ldots, x_{n}\right)^{\mathrm{T}} \in \mathbb{R}^{n}$ and all realizations $\vec{z}:=\left(z_{1}, \ldots, z_{n}\right)^{\mathrm{T}} \in \mathbb{R}^{n}$ the probability distribution of the event $\left\{\omega \in \Omega \mid \operatorname{Pr}\left(x_{1} ; \omega\right)=z_{1}, \ldots, \operatorname{Pr}\left(x_{n} ; \omega\right)=z_{n}\right\}$ is given by the multivariate Gaussian probability density

$$
\begin{aligned}
& f_{(\mu, \Sigma)}(\vec{z})=\frac{1}{\sqrt{(2 \pi)^{n} \operatorname{det}(\Sigma(\vec{x}))}} \exp \left(-\frac{1}{2}(\vec{z}-\mu(\vec{x}))^{\mathrm{T}} \Sigma(\vec{x})^{-1}(\vec{z}-\mu(\vec{x}))\right) \\
& \text { with } \mu(\vec{x}):=\left(\mathbb{E}\left[\operatorname{Pr}\left(x_{i} ; \omega\right)\right]\right)_{i=1, \ldots, n} \text { and } \Sigma(\vec{x}):=\left(\mathbb{C}\left(x_{i}, x_{j}\right)\right)_{i, j=1, \ldots, n} .
\end{aligned}
$$

Furthermore, measurements can be taken into account. Intuitively, one may think of simulating from a Gaussian distribution and then rejecting all sample paths that disagree with the measurements. Note, however, that a Gaussian process restricted to measurements is again a Gaussian process. We assume measurements $\vec{z}^{*}=\left(z_{1}^{*}, \ldots, z_{m}^{*}\right)^{\mathrm{T}} \in \mathbb{R}^{m}$ at the locations $\vec{x}^{*}=\left(x_{1}^{*}, \ldots, x_{m}^{*}\right)^{\mathrm{T}}$. Then, the conditioned process satisfies

$$
\operatorname{Pr}^{*}\left(x_{\ell}^{*} ; \omega\right):=\left.\operatorname{Pr}\left(x_{\ell}^{*} ; \omega\right)\right|_{\operatorname{Pr}\left(x^{*}\right)=z^{*}}=z_{\ell}^{*} \text { for all } \ell=1, \ldots, m .
$$

According to $\left[44\right.$, A.2], the mean and the covariance matrix of the conditioned process $\operatorname{Pr}^{*} \operatorname{read}$ as

$$
\begin{aligned}
\mu^{*}(\vec{x}) & :=\left(\mathbb{E}\left[\operatorname{Pr}^{*}\left(x_{i} ; \omega\right)\right]\right)_{i=1, \ldots, n} \\
& \left.\left.=\mu(\vec{x})+\left(\mathbb{C}\left(x_{i}, x_{\ell}^{*}\right)\right)_{\substack{i=1, \ldots, n, \ell=1, \ldots, m}}\left(\mathbb{C}\left(x_{k}^{*}, x_{\ell}^{*}\right)\right)_{\substack{k, \ell=1, \ldots, m \\
z^{*}}}^{-1} z^{*}, \operatorname{Pr}^{*}\left(x_{j} ; \omega\right)\right]\right)_{i, j=1, \ldots, n} \\
\Sigma^{*}(\vec{x}) & :=\left(\operatorname { C o v } \left[\operatorname{Pr}^{*}\left(x_{i} ; \omega\right),\left(\mathbb{C}\left(x_{i}, x_{k}^{*}\right)\right)_{\substack{i=1, \ldots, n, k=1, \ldots, m}}\left(\mathbb{C}\left(x_{k}^{*}, x_{\ell}^{*}\right)\right)_{k, \ell=1, \ldots, m}^{-1}\left(\mathbb{C}\left(x_{k}^{*}, x_{j}\right)\right)_{\substack{k=1, \ldots, m \\
j=1, \ldots, n}}\right.\right.
\end{aligned}
$$

where $\mu$ and $\mathbb{C}$ are the mean and covariance kernel of the unconditioned process (31). A widely used class of covariance kernels that generate strictly positive definite matrices $\Sigma(\vec{x})$ is the Matérn covariance function

$$
\mathbb{C}_{v}\left(x_{i}, x_{j}\right):=\sigma^{2} \frac{2^{1-v}}{\Gamma(v)}\left(\sqrt{2 v} \frac{\left|x_{i}-x_{j}\right|}{\lambda_{s}}\right)^{v} K_{v}\left(\sqrt{2 v} \frac{\left|x_{i}-x_{j}\right|}{\lambda_{s}}\right) .
$$

Here, $\Gamma$ is the gamma function, $K_{v}$ is the modified Bessel function of the second kind, $\sigma^{2}>0$ denotes the variance and $\lambda_{s}>0$ is a scaling parameter, which describes the spatial correlation of the stochastic process. The sample paths are $v-1$ times differentiable [44]. Specifically in Figure 6, we consider the

$$
\begin{aligned}
\text { exponential kernel } & \mathbb{C}_{1 / 2}(x, y)=\sigma^{2} \exp \left(-\frac{|x-y|}{\lambda_{s}}\right), \\
\text { Gaussian kernel } & \lim _{v \rightarrow \infty} \mathbb{C}_{v}(x, y)=\sigma^{2} \exp \left(-\frac{1}{2}\left(\frac{x-y}{\lambda_{s}}\right)^{2}\right) .
\end{aligned}
$$

The exponential covariance kernel describes a stationary Ornstein-Uhlenbeck process, where sample paths are not differentiable. The Gaussian kernel yields smooth sample paths. The upper panels of Figure 6 show four simulations of stress, where an exponential (left) and Gaussian (right) covariance structure is assumed as a priori distribution. The mean is plotted as dashed, black line. The 0.95 confidence region $\mathrm{CI}(x)$, satisfying $\mathbb{P}[\operatorname{Pr}(x ; \omega) \in \mathrm{CI}(x)]=0.95$, is shown in grey. The lower panels include measurements at the boundaries $x_{1}^{*}=0$ and $x_{2}^{*}=L$. As explained, the posteriori distribution that accounts for measurements 
is again a Gaussian distribution defined by $\mu^{*}$ and $\Sigma^{*}$. We observe that uncertainties are substantially decreased close to the measurements, but still mostly described by the a priori distribution in the interior of the spatial domain.
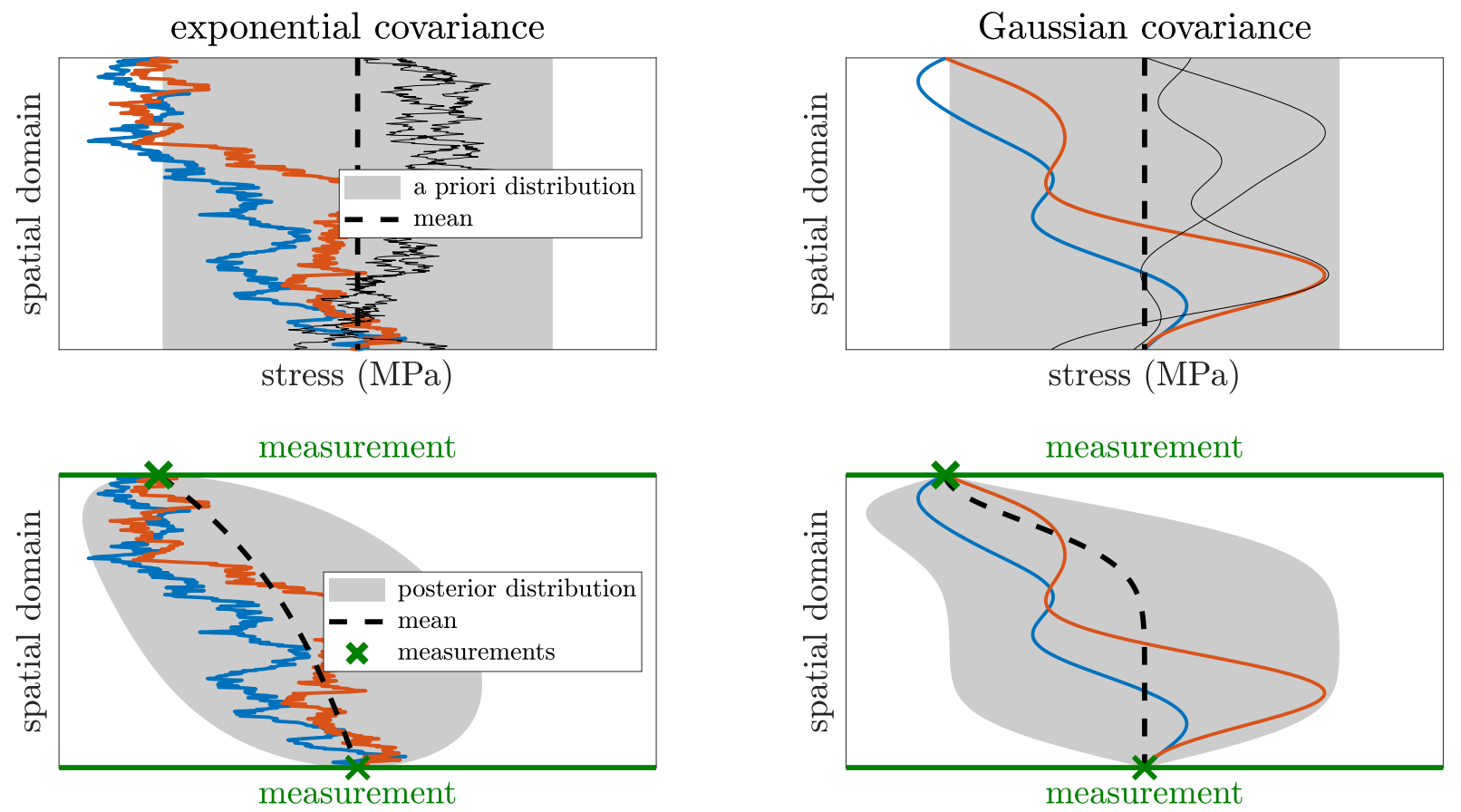

Figure 6: Upper panels show the a priori Gaussian distribution of an uncertain stress. Lower panels account for measurements (green) and show the posteriori distribution.

The eigenfunctions and eigenvalues of the strictly positive definite kernel $\mathbb{C}$ are given by the Fredholm integral equation

$$
\int_{0}^{L} \mathbb{C}(x, \cdot) \psi_{k}(x) \mathrm{d} x=d_{k} \psi_{k}(\cdot) \quad \text { with } \quad k \in \mathbb{N} .
$$

The Fredholm integral equation (FI) has been analyzed intensively. In particular, explicit solutions are known for the exponential and Gaussian kernels [44]. Furthermore, there are software packages available, for instance the chebfun-package [62], that allow for numerical solutions to general kernels. In any case, the truncation $M \in \mathbb{N}$ must be specified. Typical indicators are the explained ratio between the

$$
\text { total variance } \frac{\sum_{k=1}^{M} d_{k}}{\int_{0}^{L} \mathbb{C}(x, x) \mathrm{d} x} \text { and pointwise variance } \frac{\sum_{k=1}^{M} d_{k} \psi_{k}^{2}(x)}{\mathbb{C}(x, x)}
$$

Figure 7 illustrates these error estimates for the exponential (top) and Gaussian (bottom) covariance kernel. In each case the eigenfunctions $\psi_{1}, \ldots, \psi_{4}$ are smooth and approximate the stochastic process as superpositions in the series expansion (KL). The eigenvalues of the Gaussian kernel give more weight on the first eigenfunctions and hence, less eigenfunctions are necessary to approximate smooth sample paths. The explained total variance (32) is shown in the last panels with respect to the left vertical axis and the pointwise variance is in the scale shown at the right vertical axis. 

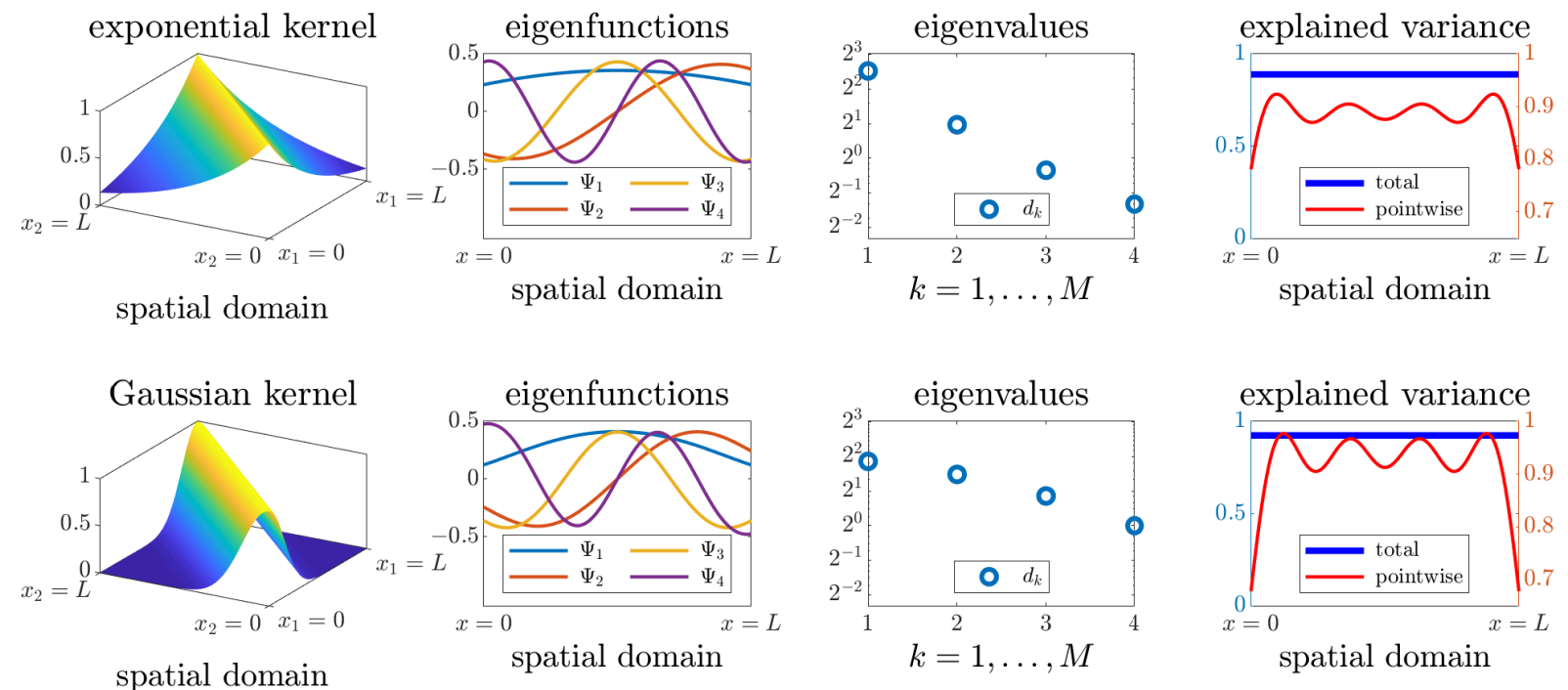

Figure 7: Karhunen-Loève decomposition for the exponential kernel (top) and Gaussian kernel (bottom).

Remark 4. The $L^{2}$-solution concept [1, Th. A.4] requires that the characteristic speeds are Lipschitz continuous and the source term must be continuous in $x \in[0, L]$. Realizations corresponding to the exponential kernel are not Lipschitz-continuous. Hence, the deterministic stability concept cannot be applied if realizations of the characteristic speeds (8) arise from a random field with exponential covariance structure. In contrast, the stability in mean squared sense may still be considered, since smooth eigenfunctions result in the truncated Karhunen-Loève expansion in smooth characteristic speeds (12). In the following we consider uncertainties in the source term, where this ambiguity does not arise.

Furthermore, the indicators (32) only state a reasonable truncation $M \in \mathbb{N}$ for the Karhunen-Loève decomposition (KL). The polynomial chaos expansion $(\mathbb{K} g \mathrm{PC})$ requires additionally a truncation $K \in \mathbb{N}$. Finding appropriate choices is subject of current research. For instance, convergence rates have been established for the linear advection equation [25], for a linear BGK model [33] and for scalar nonlinear equations [54] provided that the dependency of the solution on the stochastic input is sufficiently smooth. However, results are so far only partial and problem dependent. In the following, the choice of gPC truncation $K \in \mathbb{N}$ is not exactly justified.

\subsection{Computational results}

Using an equidistant space discretization $\Delta x>0$, the space interval $[0, L]$ is divided into $N$ cells such that $\Delta x N=L$ with cell centers $x_{i}:=\left(i-\frac{1}{2}\right) \Delta x$ for $i=1, \ldots, N$ and cell edges $x_{i+1 / 2}:=i \Delta x$ for $i=0, \ldots, N$. Additional cells with centers $x_{0}$ and $x_{N+1}$ are added outside the domain. The discrete time steps are denoted by $t_{k}:=k \Delta t$ for $k \in \mathbb{N}_{0}$ and $\Delta t>0$ such that the CFL-condition

$$
\mathrm{CFL}:=\max _{\substack{x \in[0, L],|\mathbb{K}| \\ k=1, \ldots,|\mathbb{N}|}}\left\{\widehat{\mathcal{D}}_{k}^{+}(x),\left|\widehat{\mathcal{D}}_{k}^{-}(x)\right|\right\} \frac{\Delta t}{\Delta x} \leq 1
$$

holds. Cell averages at time steps $t_{k}$ are approximated by

$$
\widehat{\zeta}_{i}^{k}:=\left(\begin{array}{l}
\widehat{\zeta}_{i}^{k,+} \\
\widehat{\zeta}_{i}^{k,-}
\end{array}\right) \approx \frac{1}{\Delta x} \int_{x_{i-1 / 2}}^{x_{i+1 / 2}}\left(\begin{array}{c}
\widehat{\zeta}^{+}\left(t_{k}, x\right) \\
\widehat{\zeta}^{-}\left(t_{k}, x\right)
\end{array}\right) \mathrm{d} x \in \mathbb{R}^{2|\mathbb{K}|} .
$$


The advection part can be approximated by a left and right sided upwind-scheme and the reaction part by the explicit Euler method, i.e.

$$
\widehat{\zeta}_{i}^{k+1}=\widehat{\zeta}_{i}^{k}-\frac{\Delta t}{\Delta x}\left(\begin{array}{cc}
\widehat{\mathcal{D}}^{+}\left(x_{i}\right) & \\
& \widehat{\mathcal{D}}^{-}\left(x_{i}\right)
\end{array}\right)\left(\begin{array}{l}
\widehat{\zeta}_{i}^{k,+}-\widehat{\zeta}_{i-1}^{k,+} \\
\hat{\zeta}_{i+1}^{k,-}-\widehat{\zeta}_{i}^{k,-}
\end{array}\right)-\Delta t \widehat{\mathcal{Q}}\left(x_{i}\right) \widehat{\zeta}_{i}^{k} \quad \text { with } \quad\left(\begin{array}{c}
\widehat{\zeta}_{0}^{k,+} \\
\widehat{\zeta}_{N+1}^{k,-}
\end{array}\right)=\widehat{\mathcal{B}}\left(\begin{array}{c}
\widehat{\zeta}_{N}^{k,+} \\
\widehat{\zeta}_{1}^{k,-}
\end{array}\right) .
$$

Since the eigenvalues $\widehat{\mathcal{D}}^{ \pm}$and the source term $\widehat{\mathcal{Q}}$ are precomputed, the computational complexity of the advection part is linear and the complexity of the source term is quadratic, since it involves a matrix-vector multiplication. Hence, computational cost is relatively low. The drawback is a restriction to a first-order method. To illustrate Theorem 2 we follow the approach in [8, Sec. 4] and approximate the continuous Lyapunov function (20) specified by the matrix (18) as

$$
\begin{aligned}
& \mathcal{L}^{k}:=\Delta x \sum_{i=1}^{N}\left(\widehat{\boldsymbol{\zeta}}_{i}^{k}\right)^{\mathrm{T}} W_{i} \widehat{\boldsymbol{\zeta}}_{i}^{k} \quad \text { with weights } W_{i}:=\operatorname{diag}\left\{W_{i}^{+}, W_{i}^{-}\right\}, \quad W_{i}^{ \pm}:=\operatorname{diag}\left\{w_{i}^{1, \pm}, \ldots, w_{i}^{|\mathbb{K}|, \pm}\right\} \\
& \text { for } \quad w_{i}^{k,+}:=\frac{h_{k}^{+}}{\widehat{\mathcal{D}}_{k}^{+}\left(x_{i}\right)} \prod_{\ell=1}^{i-1}\left(1-\Delta x \frac{\hat{\mu}}{\widehat{\mathcal{D}}_{k}^{+}\left(x_{\ell}\right)}\right) \text { and } \quad w_{i}^{k,-}:=\frac{h_{k}^{-}}{\left|\widehat{\mathcal{D}}_{k}^{-}\left(x_{i}\right)\right|} \prod_{\ell=N}^{i+1}\left(1+\Delta x \frac{\hat{\mu}}{\widehat{\mathcal{D}}_{k}^{-}\left(x_{\ell}\right)}\right)
\end{aligned}
$$

Remark 5. The continuous Lyapunov function for a single Riemann invariant with positive constant characteristic speed $\lambda>0$ is of the form

$$
\mathcal{L}(t)=\int_{0}^{L} \widehat{\zeta}(t, x)^{2} w(x) \mathrm{d} x \quad \text { for } \quad w(x):=e^{-\frac{\hat{\mu}}{\lambda} x}
$$

and a straightforward discretized analogue would be

$$
\mathcal{L}^{k}=\Delta x \sum_{i=1}^{N}\left(\widehat{\zeta}_{i}^{k}\right)^{2} e^{-\frac{\hat{\mu}}{\lambda} x_{i}}
$$

However, a blow up in the discretized derivatives occurs in contrast to the continuous case [6]. The choices (33) circumvent this problem by approximating the continuous derivative

$$
w^{\prime}(x)=-\hat{\mu} \frac{w(x)}{\lambda} \quad \text { as } \quad \frac{w_{i}-w_{i-1}}{\Delta x}=-\hat{\mu} \frac{w_{i-1}}{\lambda},
$$

where the one-sided difference quotient takes the direction of characteristic speeds into account. Then, the numerical method transfers continuous stability results to the discretized case and guarantees that also discretized Lyapunov functions decay exponentially fast over time [8, Sec. 4].

Theoretical results are illustrated for a linearized plastic part

$$
\bar{\epsilon}_{\sigma}^{p}\left(\sigma^{*}(x ; \xi(\omega))\right) \approx \mathcal{K}\left[\bar{\epsilon}_{\sigma}^{p}\left(\sigma^{*}\right)\right](x ; \omega)
$$

that is modelled by a random field with Gaussian covariance structure. Since uncertainties arise only from the source term, the transform (10) simplifies to $\widehat{\boldsymbol{\zeta}}=\widehat{\mathcal{R}}$ and physical quantities are obtained by

$\Pi_{\mathbb{K}}[y](t, x ; \xi)=\Pi_{\mathbb{K}}\left[y^{*}\right](x ; \xi)+\Pi_{\mathbb{K}}[\Delta y](t, x ; \xi) \quad$ with gPC modes $\quad \widehat{\Delta y}(t, x)=\left(\begin{array}{ll}T_{1,1}(x) \mathbb{I} & T_{1,2}(x) \mathbb{I} \\ T_{2,1}(x) \mathbb{I} & T_{2,2}(x) \mathbb{I}\end{array}\right) \widehat{\zeta}(t, x)$,

where $\mathbb{I} \in \mathbb{R}^{|\mathbb{K}| \times|\mathbb{K}|}$ denotes the identity matrix. Initial values are deterministic and stated directly in Riemann coordinates as $\mathcal{R}_{0}^{ \pm}(x)=\cos (2 \pi x)$. We choose the parameters $\mathrm{\kappa}_{0}=\mathrm{\kappa}_{1}=0.9$ in the feedback law (30). The parameters in the weights (18) are $\hat{\mu}=0.25$ and $h_{k}^{ \pm}=1$. We assume the relation $\bar{\epsilon}_{\sigma}^{p}\left(\sigma^{*}\right)=0.02 \sigma^{*}, E=100$ and as discretization we use $\Delta x=2^{-8}, L=1, \mathrm{CFL}=0.99$. The gPC basis is $\left(\mathbb{K}_{\mathrm{S}}\right)$ with Hermite polynomials and truncation $M=K=4$. 


\subsubsection{Stabilizing and destabilizing effects of the control and the source term}

The upper panels of Figure 8 are devoted to an unstable test problem. A simulation is shown, where the destabilizing effect of the source term is so strong that the applied feedback control is not sufficient to damp the introduced uncertainties. The left panel shows the mean of deviations $\mathbb{E}\left[\sigma(t, x ; \xi)-\sigma^{*}(x)\right]$ from the desired stress $\sigma^{*}=110(\mathrm{MPa})$, which increases in time. Likewise, the right panel states that the variance $\mathbb{V}[\sigma(t, x ; \xi)]$ increases exponentially fast. At the beginning there is no variance, as initial values are deterministic. But the source term introduces uncertainties over time, which are not sufficiently reduced by the boundary control. Still, the statement of Theorem 2 holds. However, the guaranteed rate $\mu \in \mathbb{R}$ is negative and hence, ensures no decay. Furthermore, we note that there are only in this test problem no deviations in the middle of the spatial domain due to the symmetric perturbation.

\section{unstable test problem}
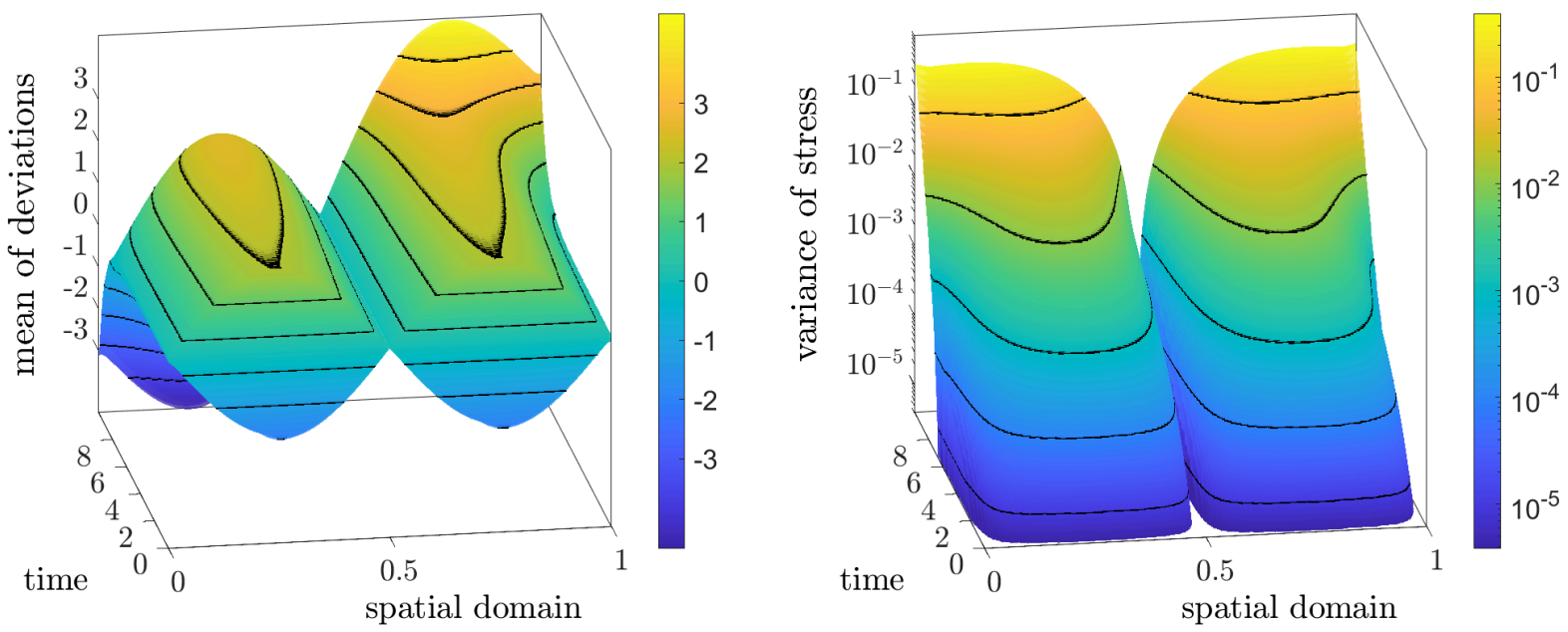

stable test problem
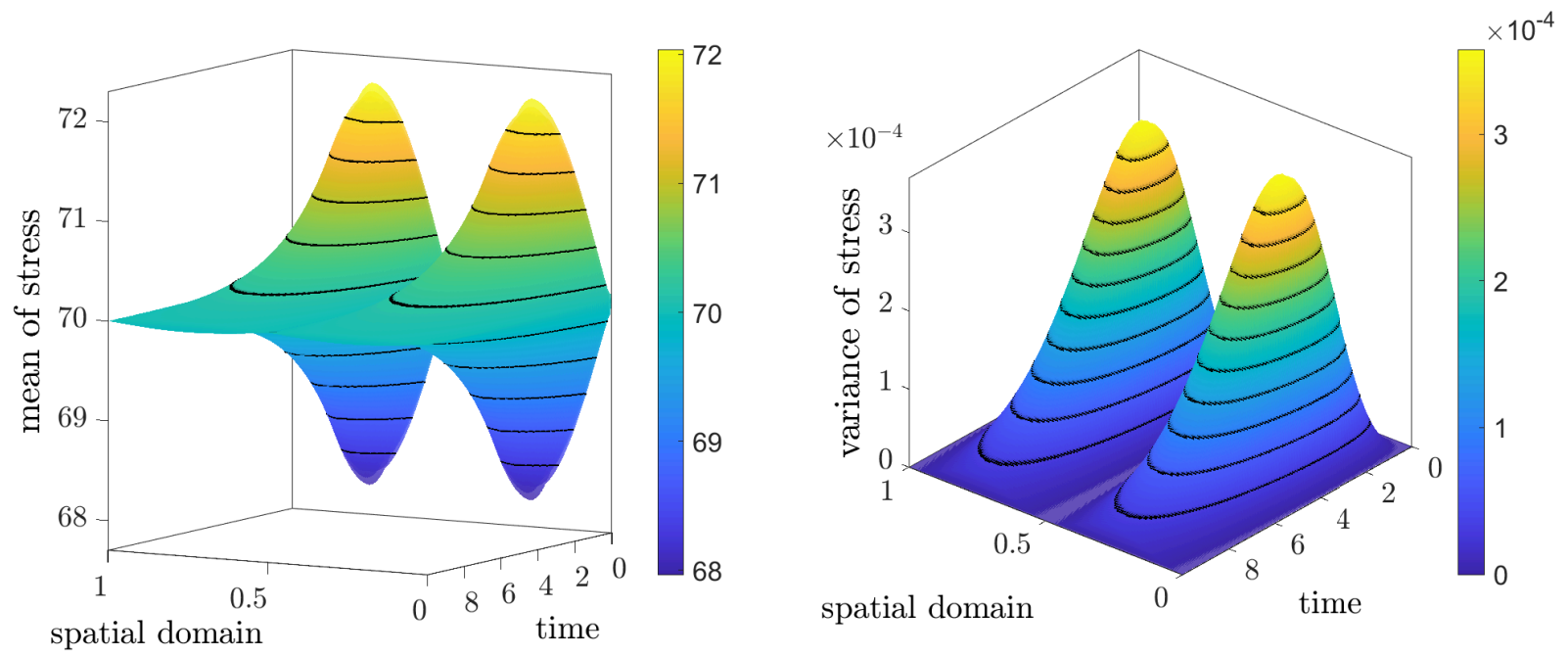

Figure 8: Unstable test problem (top): Mean of deviations $\mathbb{E}\left[\sigma(t, x ; \xi)-\sigma^{*}(x)\right]$ (left) and variance $\mathbb{V}[\sigma(t, x ; \xi)]$ (right) of uncertain stress $\sigma$ with desired state $\sigma^{*}=110(\mathrm{MPa})$. Stable test problem (bottom): Mean $\mathbb{E}[\sigma(t, x ; \xi)]$ (left) and variance $\mathbb{V}[\sigma(t, x ; \xi)]$ (right) of stress $\sigma$ with desired state $\sigma^{*}=70(\mathrm{MPa})$. 
The lower panels of Figure 8 show a simulation with desired stress $\sigma^{*}=70(\mathrm{MPa})$, where destabilizing effects of the source term are smaller. Then, the applied feedback control is sufficient to make deviations decay over time and Theorem 2 ensures a positive decay rate. The left panel shows a convergence of the mean to the desired stress $\sigma^{*}=70(\mathrm{MPa})$. At the beginning there is again no variance. Then, the variance increases, when the source term introduces uncertainties. However, the feedback law makes also the variance vanish over time. Likewise, there are no deviations in the middle of the spatial domain due to symmetric perturbations.

Figure 9 illustrates that the derived boundary control (30) allows to make the mean squared error (3) decays exponentially fast. A normalized Lyapunov function $\overline{\mathcal{L}}(t)$, which is used in the proof of Theorem 2 to make the $L^{2}$-norm $\|\widehat{\zeta}(t, \cdot)\|_{L^{2}}$ and hence the mean squared error (3) decay exponentially fast over time, is plotted in blue. The left panel shows a simulation for a stress-strain relationship described by a Bergströmmodel with state $\sigma^{*}=70(\mathrm{MPa})$ and the right panel for a DRX-model with state $\sigma^{*}=50$, respectively. As illustrated in Figure 5, the considered stress $\sigma^{*}$ is larger in the first case, which leads to a larger destabilizing effect of the source term. Hence, the feedback control steers the system faster to the desired state, when the DRX-model is used.
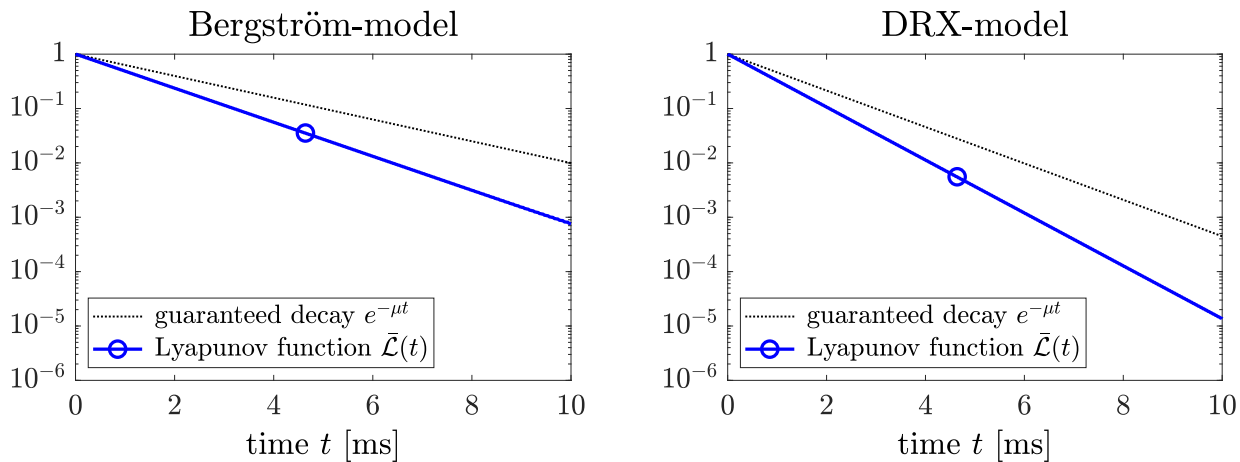

Figure 9: Normalized Lyapunov functions are plotted in blue for the feedback control (30) with parameters $\kappa_{0}=\kappa_{1}=0.9$. Theorem 2 guarantees the bound $\overline{\mathcal{L}}(t) \leq e^{-\mu t}$ (black, dotted).

\subsubsection{Numerical investigation of truncations in the polynomial chaos and Karhunen-Loève expansion}

Finally, we address the question of optimal truncations in the polynomial chaos and Karhunen-Loève expansion. Figure 10 shows for the choices $K \in\{1, \ldots, 5\}$ and $M \in\{1, \ldots, 6\}$ the observed decay rate

$$
\mu_{\mathrm{obs}}:=-\frac{1}{t_{\mathrm{end}}} \ln \left(\frac{\mathcal{L}\left(t_{\mathrm{end}}\right)}{\mathcal{L}(0)}\right)
$$

at time $t_{\text {end }}=10$. The left panel considers the stable case in Figure 8 and the right panel the unstable case. Hence, the decay rates are positive in the stable case and negative for the unstable test problem. The dashed, horizontal lines highlight the decay rates for the highest trunction $K=5$. We observe that the decay rates depend more on the number of random variables than on the degree of ansatz polynomials. Indeed, there are no changes in the rates observable for truncations $K \geq 3$. An intuitive explanation is given by the relation (15) in Remark 1 , which states that the Karhunen-Loève expansion is a special case of a gPC expansion with normally distributed random variables and truncation $K=1$. Although the input uncertainty, given by the linearized plastic part (34), requires the gPC truncation $K=1$, higher truncations are needed to expand the solution to the boundary value problem itself, since it depends on the stochastic input in a nonlinear way. Here, small truncations with $K=2$ lead to accurate results. However, larger degrees in the ansatz functions might be necessary, when the distribution is far away from being Gaussian. 
The choices $K=5$ and $M=6$ lead to a set $\left(\mathbb{K}_{\mathrm{S}}\right)$ with 462 elements. Hence, the computational results in Figure 10 also show that the discretization in Riemann coordinates, described in Section 5.3, is able to handle large systems with multiple sources of uncertainties. However, computational cost heavily depend on the truncations $(K, M)$. For the considered test problems the truncation $K=2$ seems optimal. For instance, the choice $(K, M)=(2,29)$, when the set $\left(\mathbb{K}_{\mathrm{S}}\right)$ has 465 elements. leads to a similar system size and hence to a similar computational complexity. Therefore, the investigation of an optimal truncation has here the benefit of considering 23 random variables more with the same complexity. This motivates trustful indicators that are, however, subject of further research.
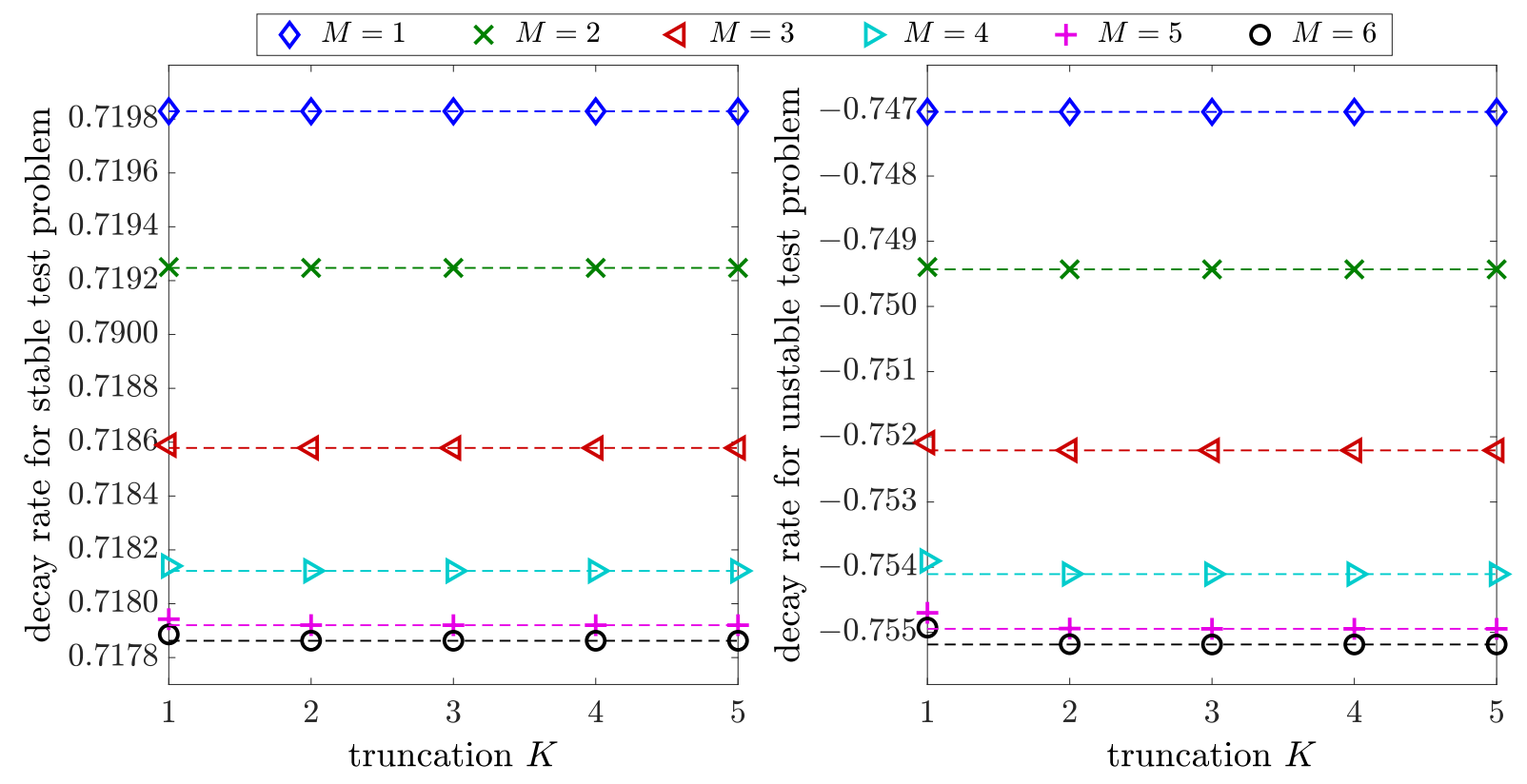

Figure 10: Comparison of truncations $K$ in the polynomial chaos expansion and number $M$ of random variables in the Karhunen-Loève expansion for the stable test problem (left) with desired stress $\sigma^{*}=70(\mathrm{MPa})$ and the unstable case with stress $\sigma^{*}=110$.

\section{SUMMARY}

We have dealt with the boundary control of random hyperbolic balance laws to damp deviations of desired states exponentially fast over time. Stochastic influences have been introduced as a series of orthogonal functions, called polynomial chaos expansions. Then, a stochastic Galerkin formulation has been derived that reformulates the underlying random system as a sequence of deterministic problems. We have established for linearized balance laws that this series comes along with countably many characteristic curves. This allows to define a Lyapunov function that yields an upper bound on mean squared deviations of a desired state. A modification of the Lyapunov stability analysis [1, Sec. 5] based on dissipative boundary conditions $[2$, Sec. 2] makes the Lyapunov function decay exponentially fast, which in turn results in a diminishing mean squared error. Furthermore, extensions and limitations to nonlinear conservation laws have been discussed.

Theoretical results have been illustrated by means of a viscoplastic material. The deforming process is partially described by a stress-strain relationship under uncertainties that are modelled by Gaussian random fields. To account for these random fields, we have described in detail the relationship between Karhunen-Loève and polynomial chaos expansions. 


\section{ACKNOWLEDGMENTS}

The authors thank the Deutsche Forschungsgemeinschaft (DFG, German Research Foundation) for the financial support through projects BA4253/11-1 and HE5386/19-1 of the priority program 2183 "PropertyControlled Forming Processes". Furthermore, this work is supported by the PRIME programme of the German Academic Exchange Service (DAAD) and we would like to offer special thanks to Simone Göttlich.

\section{References}

1. G. Bastin and J.-M. Coron. Stability and boundary stabilization of 1-d hyperbolic systems. Progress in nonlinear differential equations and their applications. Birkhäuser, Switzerland, 1 edition, 2016.

2. J.-M. Coron, G. Bastin, and B. d'Andréa-Novel. Dissipative boundary conditions for one-dimensional nonlinear hyperbolic systems. SIAM Journal on Control and Optimization, 47(3):1460-1498, 2008.

3. J.-M. Coron, G. Bastin, and B. d'Andréa-Novel. Boundary feedback control and Lyapunov stability analysis for physical networks of $2 \times 2$ hyperbolic balance laws. Proceedings of the 47 th IEEE Conference on Decision and Control, pages 1454-1458, 2008 .

4. J.-M. Coron and G. Bastin. Dissipative boundary conditions for one-dimensional quasilinear hyperbolic systems: Lyapunov stability for the $C^{1}$-norm. SIAM Journal on Control and Optimization, 53(3):1464-1483, 2015.

5. M. Gugat, G. Leugering, S. Tamasoiu, and K. Wang. $H^{2}$-stabilization of the isothermal Euler equations: A Lyapunov function approach. Chinese Annals of Mathematics, 4:479-500, 2012.

6. M. K. Banda and M. Herty. Numerical discretization of stabilization problems with boundary controls for systems of hyperbolic conservation laws. Mathematical Control and Related Fields, 3(2):121-142, 2013.

7. P. Schillen and S. Göttlich. Numerical discretization of boundary control problems for systems of balance laws: Feedback stabilization. European Journal of Control, 35:11-18, 2017.

8. S. Gerster and M. Herty. Discretized feedback control for systems of linearized hyperbolic balance laws. Mathematical Control \&3 Related Fields, 9(3):517-539, 2019.

9. V. Baumgärtner, S. Göttlich, and S. Knapp. Feedback stabilization for a coupled PDE-ODE production system. Mathematical Control \& Related Fields, 10(2):405-424, 2020.

10. M. Banda and G. Weldegiyorgis. Numerical boundary feedback stabilisation of non-uniform hyperbolic systems of balance laws. International Journal of Control, 93(6):1428-1441, 2020.

11. C. Gotzes, H. Heitsch, R. Henrion, and R. Schultz. On the quantification of nomination feasibility in stationary gas networks with random load. Mathematical Methods of Operations Research, 84(2):427-457, 2016.

12. M. Gugat and M. Schuster. Stationary gas networks with compressor control and random loads: Optimization with probabilistic constraints. Mathematical Problems in Engineering, pages 1-17, 2019.

13. N. Wiener. The homogeneous chaos. American Journal of Mathematics, 60(4):897-936, 1938.

14. R. H. Cameron and W. T. Martin. The orthogonal development of non-linear functionals in series of FourierHermite functionals. Annals of Mathematics, 48(2):385-392, 1947.

15. D. Xiu and G. E. Karniadakis. The Wiener-Askey polynomial chaos for stochastic differential equations. SIAM Journal on Scientific Computing, 24:619-644, 2002.

16. R. Shu, J. Hu, and S. Jin. A stochastic Galerkin method for the Boltzmann equation with multi-dimensional random inputs using sparse wavelet bases. Numerical Mathematics: Theory, Methods and Applications, 10(2):465488, 2017.

17. J. Hu and S. Jin. A stochastic Galerkin method for the Boltzmann equation with uncertainty. Journal of Computational Physics, 315:150-168, 2016.

18. J. Carrillo, L. Pareschi, and M. Zanella. Particle based gPC methods for mean-field models of swarming with uncertainty. Communications in Computational Physics, 25:508-531, 2019.

19. Y. Zhu and S. Jin. The Vlasov-Poisson-Fokker-Planck system with uncertainty and a one-dimensional asymptotic preserving method. Multiscale Modeling ES Simulation, 15:1502-1529, 2017. 
20. S. Jin and Y. Zhu. Hypocoercivity and uniform regularity for the Vlasov-Poisson-Fokker-Planck system with uncertainty and multiple scales. SIAM Journal on Mathematical Analysis, 50(2):1790-1816, 2018.

21. J. Carrillo and M. Zanella. Monte Carlo gPC methods for diffusive kinetic flocking models with uncertainties. Vietnam Journal of Mathematics, 47:931-954, 2019.

22. M. Zanella. Structure preserving stochastic Galerkin methods for Fokker-Planck equations with background interactions. Mathematics and Computers in Simulation, 168:28-47, 2020.

23. B. Després, G. Poëtte, and D. Lucor. Uncertainty quantification for systems of conservation laws. Journal of Computational Physics, 228:2443-2467, 2009.

24. Q.-Y. Chen, D. Gottlieb, and J. S. Hesthaven. Uncertainty analysis for the steady-state flows in a dual throat nozzle. Journal of Computational Physics, 204:378-398, 2005.

25. D. Gottlieb and D. Xiu. Galerkin method for wave equations with uncertain coefficients. Communications in Computational Physics, 3(2):505-518, 2008.

26. R. Pulch and D. Xiu. Generalised polynomial chaos for a class of linear conservation laws. Journal of Scientific Computing, 51:293-312, 2012.

27. P. Pettersson, G. Iaccarino, and J. Nordström. A stochastic Galerkin method for the Euler equations with Roe variable transformation. Journal of Computational Physics, 257:481-500, 2014.

28. S. Gerster, M. Herty, and A. Sikstel. Hyperbolic stochastic Galerkin formulation for the p-system. Journal of Computational Physics, 395:186-204, 2019.

29. S. Gerster and M. Herty. Entropies and symmetrization of hyperbolic stochastic Galerkin formulations. Communications in Computational Physics, 27:639-671, 2020.

30. J. Kusch, G. Alldredge, and M. Frank. Maximum-principle-satisfying second-order intrusive polynomial moment scheme. SMAI Journal of Computational Mathematics, 5:23-51, 2017.

31. Q. Li and L. Wang. Uniform regularity for linear kinetic equations with random input based on hypocoercivity. SIAM Journal on Uncertainty Quantification, 5:1193-1219, 2017.

32. L. Liu and J. Shi. Hypocoercivity based sensitivity analysis and spectral convergence of the stochastic Galerkin approximation to collisional kinetic equations with multiple scales and random inputs. Multiscale Modeling $\varepsilon$ Simulation, 16(3):1085-1114, 2018.

33. S. Gerster, M. Herty, and H. Yu. Hypocoercivity of stochastic Galerkin formulations for stabilization of kinetic equations. Communications in Mathematical Sciences, 19(3):787-806, 2021.

34. F. Hérau. Hypocoercivity and exponential time decay for the linear inhomogeneous relaxation Boltzmann equation. Asymptotic Analysis, 46:349-359, 2006.

35. F. Hérau. Introduction to hypocoercive methods and applications for simple linear inhomogeneous kinetic models. In Lectures on the Analysis of Nonlinear Partial Differential Equations, volume 5 of Morning Side Lectures in Mathematics Series, pages 119-147. 2017.

36. C. Villani. Hypocoercivity, volume 202 of Memoirs of the American Mathematical Society. Providence, RI, 2009.

37. J. Dolbeault, C. Mouhot, and C. Schmeiser. Hypocoercivity for kinetic equations with linear relaxation terms. Comptes Rendus Mathematique, 347:511-516, 2009.

38. J. Dolbeault, C. Mouhot, and C. Schmeiser. Hypocoercivity for linear kinetic equations conserving mass. Transactions of the American Math. Society, 367:3807-3828, 2015.

39. C. M. Dafermos. Hyperbolic conservation laws in continuum physics, volume 325 of A series of comprehensive studies in mathematics. Springer-Verlag Berlin Heidelberg, 3 edition, 2010.

40. H. K. Khalil. Nonlinear control. Pearson Education, 2015.

41. G. Bastin and J.-M. Coron. On boundary feedback stabilization of non-uniform linear $2 \times 2$ hyperbolic systems over a bounded interval. Systems \& Control Letters, 60(11):900-906, 2011.

42. M. Gugat and S. Gerster. On the limits of stabilizability for networks of strings. Systems 85 Control Letters, 131:1-10, 2019.

43. J. Nordström and M. Wahlsten. Variance reduction through robust design of boundary conditions for stochastic hyperbolic systems of equations. Journal of Computational Physics, 282:1-22, 2015. 
44. C. E. Rasmussen and C. K. I. Williams. Gaussian Processes for Machine Learning (Adaptive Computation and Machine Learning). The MIT Press, 2 edition, 2005.

45. O. P. Le Maître and O. M. Knio. Spectral Methods for uncertainty quantification. Springer Netherlands, 1 edition, 2010.

46. D. Funaro. Polynomial approximation of differential equations, volume 8. Springer Science \& Business Media, 2008.

47. R. Courant and D. Hilbert. Methods of Mathematical Physics, volume 1. Wiley, New York, 1989.

48. O. G. Ernst, A. Mugler, H. J. Starkloff, and E. Ullmann. On the convergence of generalized polynomial chaos expansions. ESAIM: M2AN, 46(2):317-339, 2012.

49. B. Sonday, R. Berry, H. Najm, and B. Debusschere. Eigenvalues of the Jacobian of a Galerkin-projected uncertain ODE system. Journal of Scientific Computing, 33:1212-1233, 2011.

50. D. Xiu. Numerical methods for stochastic computations. Princeton University Press, Princeton, 2010.

51. D. Xiu and G. E. Karniadakis. Supersensitivity due to uncertain boundary conditions. International Journal for Numerical Methods in Engineering, 61:2114-2138, 2004.

52. B. Riemann. Über die Fortpflanzung ebener Luftwellen von endlicher Schwingungsweite. Abhandlungen der Königlichen Gesellschaft der Wissenschaften in Göttingen, 8:43-66, 1860.

53. D. Dai, Y. Epshteyn, and A. Narayan. Hyperbolicity-preserving and well-balanced stochastic Galerkin method for shallow water equations. SIAM Journal on Scientific Computing, 43(2):A929-A952, 2021.

54. B. Després, G. Poëtte, and D. Lucor. Robust uncertainty propagation in systems of conservation laws with the entropy closure method, volume 92 of Uncertainty Quantification in Computational Fluid Dynamics. Lecture Notes in Computational Science and Engineering. Springer, Cham, 2013.

55. P. D. Lax. Hyperbolic Systems of Conservation Laws and the Mathematical Theory of Shock Waves. Society for Industrial and Applied Mathematics, 1973.

56. L. Ta-Tsien, Z. Yi, and K. De-Xing. Global classical solutions for general quasilinear hyperbolic systems with decay initial data. Nonlinear Analysis: Theory, Methods $\&$ Applications, 28(8):1299-1332, 1997.

57. S. Jin, D. Xiu, and X. Zhu. A well-balanced stochastic Galerkin method for scalar hyperbolic balance laws with random inputs. Journal of Scientific Computing, 67:1198-1218, 2016.

58. R. J. Leveque. Finite volume methods for hyperbolic problems. Cambridge Texts in Applied Mathematics. Cambridge University Press, 1 edition, 2002.

59. J. C. Simo and T. J. R. Hughes. Computational Inelasticity. Springer, New York, 1 edition, 2016.

60. Y. Bergström. A dislocation model for the stress-strain behaviour of polycrystalline $\alpha$-Fe with special emphasis on the variation of the densities of mobile and immobile dislocations. Materials Science and Engineering, 5(4):193-200, 1970.

61. M. Bambach. Implications from the Poliak-Jonas criterion for the construction of flow stress models incorporating dynamic recrystallization. Acta materialia, 61(16):6222-6233, 2013.

62. T. A. Driscoll, N. Hale, and L. N. Trefethen. Chebfun guide. Pafnuty Publications, Oxford, 2014. 